Pacific Journal of Mathematics

WORD EQUATIONS IN SOME GEOMETRIC SEMIGROUPS 


\title{
WORD EQUATIONS IN SOME GEOMETRIC SEMIGROUPS
}

\author{
Mohan S. Putcha
}

Let $S$ be a semigroup and let $w_{1}=w_{1}\left(x_{1}, \cdots, x_{t}\right), w_{2}=$ $w_{2}\left(x_{1}, \cdots, x_{t}\right)$ be two words in the variables $x_{1}, \cdots, x_{t \bullet} \quad$ By a solution of the word equation $\left\{w_{1}, w_{2}\right\}$ in $S$, we mean $a_{1}, \cdots, a_{t} \in$ $S$ such that $w_{1}\left(a_{1}, \cdots, a_{t}\right)=w_{2}\left(a_{1}, \cdots, a_{t}\right)$. Let $\mathscr{F}_{\mathrm{R}}$ denote the free product of $t$ copies of positive reals under addition. In $\$ 3$ and $\$ 5$ we show that if $Y$ is either the semigroup of certain paths in $\mathbf{R}^{\boldsymbol{n}}$ or the semigroup of designs around the unit disc, then any solution of $\left\{w_{1}, w_{2}\right\}$ in $Y$ can be derived from a solution of $\left\{w_{1}, w_{2}\right\}$ in $\mathscr{F}_{\mathbf{R}}$. This answers affirmatively a problem posed in Word equations of paths by Putcha. Word equations in $\mathscr{F}_{\mathrm{R}}$ are studied in $\$ 1$. Using these results, it is shown that any solution in $Y$ of $\left\{w_{1}, w_{2}\right\}$ can be approximated by a solution which is derived from a solution in a free semigroup. There are two books by Hmelevskii and Lentin on word equations in free semigroups. We also show that if $\left\{w_{1}, w_{2}\right\}$ has only trivial solutions in any free semigroup, then it has only trivial solutions in $Y$.

1. Preliminaries. Throughout this paper, $\mathbf{N}, Z, Z^{+}, 2,2^{+}, \mathbf{R}$, $\mathbf{R}^{+}$will denote the sets of nonnegative integers, integers, positive integers, rationals, positive rationals, reals and positive reals, respectively. For $m, n \in Z^{+}$, let $\mathbf{R}^{m \times n}, 2^{m \times n}$ denote the sets of all $m \times n$ matrices over the reals and rationals, respectively. If $S$ is a semigroup, then $S^{1}=S \cup\{1\}$ with obvious multiplication if $S$ does not have an identity element; $S^{1}=S$ otherwise. If $T \subseteq S^{1}$, then $T^{1}=T \cup\{1\}$.

Definition. Let $S$ be a semigroup and $a, b \in S$.

(1) $a \mid b$ if $b=x a y$ for some $x, y \in S^{1}$.

(2) $\left.a\right|_{i} b$ if $b=a x$ for some $x \in S^{1}$.

(3) $\left.a\right|_{f} b$ if $b=y a$ for some $y \in S^{1}$.

If $\Gamma$ is a nonempty set, then let $\mathscr{F}=\mathscr{F}(\Gamma)$ denote the free semigroup on $\Gamma$. If $w \in \mathscr{F}$, then let $l(w)=$ length of $w$. If $S$ is a semigroup and $a_{1}, \cdots, a_{n} \in S$, then we say that $a \in S$ is a word in $a_{1}, \cdots, a_{n}$ if $a=$ $w\left(a_{1}, \cdots, a_{n}\right)$ for some $w\left(x_{1}, \cdots, x_{n}\right) \in \mathscr{F}\left(x_{1}, \cdots, x_{n}\right)$. This is the same as saying that $a$ is an element of the semigroup generated by $a_{1}, \cdots, a_{n}$.

Let $\Gamma$ be a nonempty set. Let $\mathscr{F}_{\mathbf{R}}=\mathscr{F}_{\mathbf{R}}(\Gamma)$ denote the set of all nonempty finite sequences (also called words) of the type $w=A_{1}^{\alpha_{1}} \cdots A_{n}^{\alpha_{n}}$ 
where $n \in Z^{+}, \alpha_{1}, \cdots, \alpha_{n} \in \mathbf{R}^{+}, A_{1}, \cdots, A_{n} \in \Gamma$ and $A_{\imath} \neq A_{i+1}$ for $i, i+1 \in$ $\{1, \cdots, n\}$. We define $e(w)=n$ and $l(w)=\alpha_{1}+\cdots+\alpha_{n}$. Let $w_{1}, w_{2} \in \mathscr{F}_{\mathbf{R}}$. Suppose $w_{1}=A_{1}^{\alpha_{1}} \cdots A_{n}^{\alpha_{n}}, \quad w_{2}=B_{1}^{\beta_{1}} \cdots B_{m}^{\beta_{m}}$. Then we define

$$
w_{1} w_{2}=\left\{\begin{array}{lll}
A_{1}^{\alpha_{1}} \cdots A_{n}^{\alpha_{n}+\beta_{1}} B_{2}^{\beta_{2}} \cdots B_{m}^{\beta_{m}} & \text { if } & A_{n}=B_{1} . \\
A_{1}^{\alpha_{1}} \cdots A_{n}^{\alpha_{n}} B_{1}^{\beta_{1}} \cdots B_{m}^{\beta_{m}} & \text { if } & A_{n} \neq B_{1} .
\end{array}\right.
$$

Now, of course, expressions of the type $w=A_{1}^{\alpha_{1}} \cdots A_{n}^{\alpha_{n}}\left(\alpha_{1}, \cdots, \alpha_{n} \in \mathbf{R}^{+}\right.$; $\left.A_{1}, \cdots, A_{n} \in \Gamma\right)$ make sense even when $A_{i}=A_{i+1}$ for some $i, i+1 \in$ $\{1, \cdots, n\}$. But note that if $n=e(w)$, then $A_{i} \neq A_{i+1}$ for any $i, i+1 \in$ $\{1, \cdots, n\}$. In such a case we call $\boldsymbol{A}_{1}^{\alpha_{1}} \cdots A_{n}^{\alpha_{n}}$, the standard form of $w$. $\mathscr{F}_{\mathbf{R}}(\Gamma)$ is a semigroup and is just the free product of $|\Gamma|$ copies of $\mathbf{R}^{+}$ under addition (see for example $[3 ;$ p. 411]). Let $\mathcal{N}=\mathcal{N}(\Gamma)=$ $\left\{A^{\alpha} \mid A \in \Gamma, \alpha \in \mathbf{R}^{+}\right\}$. If $u, v \in \mathscr{F}_{\mathbf{R}}(\Gamma)$, then define $u \sim v$ if either $u=w^{\prime}$, $v=w^{\prime}$ for some $w \in \mathscr{F}_{\mathbf{R}}, i, j \in Z^{+}$or if $u=A^{\alpha}, v=A^{\beta}$ for some $\alpha, \beta \in \mathbf{R}^{+}, A \in \Gamma$. Clearly, $\sim$ is an equivalence relation on $\mathcal{N}(\Gamma)$. It will follow from Theorem 1.9 that $\sim$ is in fact an equivalence relation on $\mathscr{F}_{\mathbf{R}}(\Gamma)$. Let $w \in \mathscr{F}_{\mathbf{R}}, w=A_{1}^{\alpha_{1}} \cdots A_{n}^{\alpha_{n}}$ in standard form. Let $A \in \Gamma$. Then $A$ appears integrally in $w$ if for each $i \in\{1, \cdots, n\}, A_{i}=A$ implies $\alpha_{i} \in Z^{+}$. Otherwise $A$ appears nonintegrally in $w$. $A$ appears rationally in $w$ if for each $i \in\{1, \cdots, n\}, A_{i}=A$ implies $\alpha_{i} \in 2^{+}$. Let $\mathscr{F}_{2}(\Gamma)=\left\{w \mid w \in \mathscr{F}_{\mathbf{R}}(\Gamma), A\right.$ appears rationally in $w$ for each $A \in$ $\Gamma\} . \quad \mathscr{F}_{2}(\Gamma)$ is a subsemigroup of $\mathscr{F}_{\mathbf{R}}(\Gamma)$.

Definition. By a word equation in variables $x_{1}, \cdots, x_{n}$ we mean $\left\{w_{1}, w_{2}\right\}$ where $w_{1}=w_{1}\left(x_{1}, \cdots, x_{n}\right), w_{2}=w_{2}\left(x_{1}, \cdots, x_{n}\right) \in \mathscr{F}\left(x_{1}, \cdots, x_{n}\right)$. It is not necessary that each $x_{\imath}$ appears in $w_{1} w_{2}$. Let $S$ be a semigroup and $a_{1}, \cdots, a_{n} \in S$. Then $\left(a_{1}, \cdots, a_{n}\right)$ is a solution of $\left\{w_{1}, w_{2}\right\}$ if $w_{1}\left(a_{1}, \cdots, a_{n}\right)=w_{2}\left(a_{1}, \cdots, a_{n}\right)$.

Let $\left(b_{1}, \cdots, b_{n}\right)$ be a solution in $\mathscr{F}(\Gamma)$ of a word equation $\left\{w_{1}, w_{2}\right\}$ in variables $x_{1}, \cdots, x_{n}$. Let $S$ be a semigroup and $\varphi: \mathscr{F}(\Gamma) \rightarrow S$, a homomorphism. Let $a_{i}=\varphi\left(b_{i}\right), i=1, \cdots, n$. Then $\left(a_{1}, \cdots, a_{n}\right)$ is a solution of $\left\{w_{1}, w_{2}\right\}$. We say that $\left(a_{1}, \cdots, a_{n}\right)$ follows from $\left(b_{1}, \cdots, b_{n}\right)$.

Definition. Let $\left\{w_{1}, w_{2}\right\}$ be a word equation in variables $x_{1}, \cdots, x_{n}$ and $S$ a semigroup.

(1) Let $\left(a_{1}, \cdots, a_{n}\right)$ be a solution of $\left\{w_{1}, w_{2}\right\}$ in $S$. Then $\left(a_{1}, \cdots, a_{n}\right)$ is strongly resolvable if it follows from some solution of $\left\{w_{1}, w_{2}\right\}$ in $\mathscr{F}(\Gamma)$ for some nonempty set $\Gamma$. By Lentin [2] we can then choose $|\Gamma| \leqq n$.

(2) $\left\{\boldsymbol{w}_{1}, \boldsymbol{w}_{2}\right\}$ is strongly resolvable in $S$ if every solution of $\left\{\boldsymbol{w}_{1}, \boldsymbol{w}_{2}\right\}$ is strongly resolvable. 
Let $\Gamma$ be a nonempty set and let $\xi: \Gamma \rightarrow 2^{+}$. Then clearly there exists a unique automorphism $\varphi$ of $\mathscr{F}_{2}(\Gamma)$ such that $\varphi(A)=A^{\xi(A)}$ for all $A \in \Gamma$. Now let $a_{1}, \cdots, a_{n} \in \mathscr{F}_{2}(\Gamma)$. Then there exists an automorphism $\varphi$ of $\mathscr{F}_{2}(\Gamma)$ of the above type such that $b_{l}=\varphi\left(a_{l}\right) \in \mathscr{F}(\Gamma)$, $i=1, \cdots, n$. Suppose $\left(a_{1}, \cdots, a_{n}\right)$ is a solution of a word equation. Then $\left(b_{1}, \cdots, b_{n}\right)$ is also a solution of the same equation and $a_{i}=\varphi^{-1}\left(b_{i}\right), i=1, \cdots, n$. So we have the following.

THEOREM 1.1. Every word equation is strongly resolvable in $\mathscr{F}_{2}(\Gamma)$ for any nonempty set $\Gamma$.

Definition. Let $w_{1}, w_{2} \in \mathscr{F}_{\mathbf{R}}(\Gamma)$. Suppose $w_{1}=A_{1}^{\alpha_{1}} \cdots A_{n}^{\alpha_{n}}, w_{2}=$ $B_{1}^{\beta_{1}} \cdots B_{m}^{\beta_{m}}$ in standard form. If $m=n$ and $A_{l}=B_{\imath}(i=1, \cdots, n)$, then let $d\left(w_{1}, w_{2}\right)=\sum_{i=1}^{n}\left|\alpha_{i}-\beta_{i}\right|$. Otherwise let $d\left(w_{1}, w_{2}\right)=\infty$.

LEMMA 1.2. Let $u_{1}, u_{2}, u_{3}, u_{4} \in \mathscr{F}_{\mathbf{R}}(\Gamma)$. Then the following are true in the extended real line.

(i) $e\left(u_{1} u_{2}\right)=e\left(u_{1}\right)+e\left(u_{2}\right)$ or $e\left(u_{1}\right)+e\left(u_{2}\right)-1$.

(ii) $d\left(u_{1}, u_{2}\right)=0$ if and only if $u_{1}=u_{2}$.

(iii) $d\left(u_{1}, u_{3}\right) \leqq d\left(u_{1}, u_{2}\right)+d\left(u_{2}, u_{3}\right)$.

(iv) $d\left(u_{1}, u_{2}\right)=d\left(u_{2}, u_{1}\right)$.

(v) $d\left(u_{1} u_{2}, u_{3} u_{4}\right) \leqq d\left(u_{1}, u_{3}\right)+d\left(u_{2}, u_{4}\right)$.

Proof. (i), (ii), (iii) and (iv) are clear. So we prove (v). Let $w_{1}, w_{2} \in \mathscr{F}_{\mathbf{R}}(\Gamma), d\left(w_{1}, w_{2}\right)<\infty$. Let $w_{1}=A_{1}^{\alpha_{1}} \cdots A_{n}^{\alpha_{n}}, w_{2}=A_{1}^{\beta_{1}} \cdots A_{n}^{\beta_{n}}$ in standard form. Let $A \in \Gamma$. If $A \neq A_{n}$, then for any $\alpha \in \mathbf{R}^{+}, w_{1} A^{\alpha}=$ $A_{1}^{\alpha_{1}} \cdots A_{n}^{\alpha_{n}} A^{\alpha}, \quad w_{2} A^{\alpha}=A_{1}^{\beta_{1}} \cdots A_{n}^{\beta_{n}} A^{\alpha} \quad$ in standard form. So $d\left(w_{1} A^{\alpha}, w_{2} A^{\alpha}\right)=d\left(w_{1}, w_{2}\right)$. If $A=A_{n}$, then $w_{1} A^{\alpha}=A_{1}^{\alpha_{1}} \cdots A_{n}^{\alpha_{n}+\alpha}$, $w_{2} A^{\alpha}=A_{1}^{\beta_{1}} \cdots A_{n}^{\beta_{n}+\alpha}$. So again $d\left(w_{1} A^{\alpha}, w_{2} A^{\alpha}\right)=d\left(w_{1}, w_{2}\right)$. So by induction $\quad d\left(w_{1} u, w_{2} u\right)=d\left(w_{1}, w_{2}\right)$ for all $u \in \mathscr{F}_{\mathbf{R}}(\Gamma)$. Similarly $d\left(u w_{1}, u w_{2}\right)=d\left(w_{1}, w_{2}\right)$ for all $u \in \mathscr{F}_{\mathbf{R}}(\Gamma)$. Let $u_{1}, u_{2}, u_{3}, u_{4} \in \mathscr{F}_{\mathbf{R}}(\Gamma)$ such that $d\left(u_{1}, u_{3}\right)<\infty$ and $d\left(u_{2}, u_{4}\right)<\infty$. So $d\left(u_{1} u_{2}, u_{3} u_{4}\right) \leqq$ $d\left(u_{1} u_{2}, u_{3} u_{2}\right)+d\left(u_{3} u_{2}, u_{3} u_{4}\right)=d\left(u_{1}, u_{3}\right)+d\left(u_{2}, u_{4}\right)$. The same holds trivially if $d\left(u_{1}, u_{3}\right)=\infty$ or $d\left(u_{2}, u_{4}\right)=\infty$.

LeMmA 1.3. (i) Let $u \in \mathscr{F}_{\mathbf{R}}(\Gamma), n \in Z^{+}$such that $e(u)>1$. Let $u=A_{1}^{\alpha_{1}} \cdots A_{r}^{\alpha_{r}}, u^{n}=B_{1}^{\beta_{1}} \cdots B_{s}^{\beta_{s}}$ in standard form. Then $\left\{\alpha_{1}, \cdots, \alpha_{r}\right\} \subseteq$ $\left\{\beta_{1}, \cdots, \beta_{s}\right\}$.

(ii) Let $u, v \in \mathscr{F}_{\mathbf{R}}(\Gamma), n \in Z^{+}$. Then $d(u, v) \leqq d\left(u^{n}, v^{n}\right) \leqq n d(u, v)$.

Proof. (i) $1<r \leqq s$. Since $\left.u\right|_{i} u^{n},\left.\quad u\right|_{f} u^{n}$ we obtain $\alpha_{t}=\beta_{\imath}$ $(1 \leqq i<r)$ and $\alpha_{r}=\beta_{s}$.

(ii) That $d\left(u^{n}, v^{n}\right) \leqq n d(u, v)$ follows from Lemma $1.2(\mathrm{v})$. So we 
show that $d(u, v) \leqq d\left(u^{n}, v^{n}\right)$. If $d\left(u^{n}, v^{n}\right)=\infty$, this is trivial. So let $d\left(u^{n}, v^{n}\right)<\infty$. If $u^{n}$ or $v^{n} \in \mathcal{N}(\Gamma)$, then $u, v \in \mathcal{N}(\Gamma)$ and $u \sim v$. So for some $A \in \Gamma, \quad \epsilon, \delta \in \mathbf{R}^{+}, \quad u=A^{\epsilon}, \quad v=A^{\delta}$. So $d(u, v)=|\epsilon-\delta| \leqq$ $|n \epsilon-n \delta|=d\left(u^{n}, v^{n}\right)$. Next assume $e\left(u^{n}\right), e\left(v^{n}\right)>1$. Let $u^{n}=$ $A_{1}^{\alpha_{1}} \cdots A_{m}^{\alpha_{m}}, v^{n}=A_{1}^{\beta_{1}} \cdots A_{m}^{\beta_{m}}$ in standard form with $m>1$. Let $u=$ $B_{1}^{\gamma_{1}} \cdots B_{r}^{\gamma_{r}}, v=C_{1}^{\delta_{1}} \cdots C_{s}^{\delta_{s}}$ in standard form. Then $r, s>1, B_{1}=A_{1}=$ $C_{1}, B_{r}=A_{m}=C_{s}$. If $A_{1} \neq A_{m}$, then $r n=m=s n$. So $r=s$. If $A_{1}=$ $A_{m}$, then $r-n-1=m=n s-n-1$. Thus in any case $r=s$. Also $B_{i}=A_{\imath}=C_{l}, \quad 1 \leqq i \leqq r$. For $1 \leqq i \leqq r-1, \gamma_{i}=\alpha_{t}$ and $\delta_{i}=\beta_{i}$. Also $\gamma_{r}=\alpha_{m}$ and $\delta_{s}=\beta_{m}$. Thus $\sum_{t=1}^{r}\left|\gamma_{i}-\delta_{i}\right| \leqq \sum_{i=1}^{m}\left|\alpha_{t}-\beta_{l}\right|$. This proves the lemma.

If $P \in \mathbf{R}^{m \times n}$, then let $P^{T}$ denote the transpose of $P$.

Lemma 1.4. Let $\Gamma$ be a nonempty set and let $A_{1}, \cdots, A_{n} \in \Gamma$, $\epsilon_{1}, \cdots, \epsilon_{n} \in \mathbf{R}^{+}, i_{1}, \cdots, i_{r}, j_{1}, \cdots, j_{s} \in\{1, \cdots, n\}$. Suppose that in $\mathscr{F}_{\mathbf{R}}(\Gamma)$,

$$
A_{{ }_{11}}^{\epsilon_{i 1}} \cdots A_{i_{r}}^{\epsilon_{i,}}=A_{j_{1}}^{\epsilon_{1}} \cdots A_{l_{s}}^{\epsilon_{t_{s}}} .
$$

Then there exists $P \in 2^{m \times n}$ for some $m \in Z^{+}$such that for any $\alpha_{1}, \cdots, \alpha_{n} \in$ $\mathbf{R}^{+}, P\left(\alpha_{1}, \cdots, \alpha_{n}\right)^{T}=0$ if and only if

$$
A_{t_{1}, \cdots}^{\alpha_{i}} \cdots A_{l_{r}}^{\alpha_{t r}}=A_{j 1}^{\alpha_{1}} \cdots A_{l_{s}}^{\alpha_{l_{s}}}
$$

Proof. We prove by induction on $r+s$. Choose $p, q$ maximal so that $1 \leqq p \leqq r, 1 \leqq q \leqq s$ and for any $\alpha, \beta$ with $1 \leqq \alpha \leqq p, 1 \leqq \beta \leqq q$, we have $A_{i 1}=A_{i_{\alpha}}$ and $A_{11}=A_{j \beta}$. Clearly $A_{i_{1}}=A_{11}$ and $\sum_{k=1}^{p} \epsilon_{i k}=$ $\sum_{k=1}^{q} \epsilon_{l k}$. Now clearly $p=r$ if and only if $q=s$. Also in this case, for any $\alpha_{1}, \cdots, \alpha_{n} \in \mathbf{R}^{+}$, (1) holds if and only if $\sum_{k=1}^{r} \alpha_{t k}=\sum_{k=1}^{s} \alpha_{j k}$. We can then trivially choose a $1 \times n$ integer matrix $P$ such that for any $\alpha_{1}, \cdots, \alpha_{n} \in \mathbf{R}^{+}$, $P\left(\alpha_{1}, \cdots, \alpha_{n}\right)^{T}=0$ if and only if $\sum_{k=1}^{r} \alpha_{l k}=\sum_{k=1}^{s} \alpha_{j k}$.

Thus we may assume $p<r$ adn $q<s$. Then we have

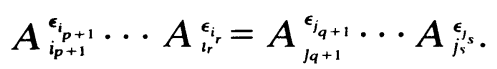

If $\alpha_{1}, \cdots, \alpha_{n} \in \mathbf{R}^{+}$, then (1) holds if and only if

$$
\sum_{k=1}^{p} \alpha_{i k}=\sum_{k=1}^{q} \alpha_{j k}
$$

and

$$
A_{i_{p}+1}^{\alpha_{i_{p}+1}} \cdots A_{t_{r}}^{\alpha_{i_{r}}}=A_{j_{q+1}}^{\alpha_{l_{q}+1}} \cdots A_{l_{s}}^{\alpha_{t_{s}}} .
$$


We can trivially choose a $1 \times n$ integer matrix $P_{1}$ such that (2) holds if and only if $P_{1}\left(\alpha_{1}, \cdots, \alpha_{n}\right)^{T}=0$. By our induction hypothesis, we can choose $P_{2} \in 2^{m \times n}$ for some $m$ such that (3) holds if and only if $P_{2}\left(\alpha_{1}, \cdots, \alpha_{n}\right)^{T}=$ 0 . Let $P=\left(\begin{array}{l}P_{1} \\ P_{2}\end{array}\right)$. Then for any $\alpha_{1}, \cdots, \alpha_{n} \in \mathbf{R}^{+}, P\left(\alpha_{1}, \cdots, \alpha_{n}\right)^{T}=0$ if and only if both (2) and (3) hold. This proves the lemma.

Lemma 1.5. Let $\Gamma$ be a nonempty set and let $A_{1}, \cdots, A_{n} \in \Gamma$, $\epsilon_{1}, \cdots, \epsilon_{n} \in \mathbf{R}^{+}, i_{1}, \cdots, i_{r}, j_{1}, \cdots, j_{s} \in\{1, \cdots, n\}$. Suppose that in $\mathscr{F}_{\mathbf{R}}(\Gamma)$,

$$
A_{l_{1}}^{\epsilon_{1}} \cdots A_{t_{r}}^{\epsilon_{i r}}=A_{j_{1}}^{\epsilon_{1}} \cdots A_{f_{i s}}^{\epsilon_{i_{s}}}
$$

Let $\delta \in \mathbf{R}^{+}$. Then there exist $\alpha_{1}, \cdots, \alpha_{n} \in 2^{+}$such that $\sum_{k=1}^{n}\left|\alpha_{k}-\epsilon_{k}\right|<\delta$ and

$$
A_{i_{1}}^{\alpha_{11}} \cdots A_{l_{r}}^{\alpha_{r}^{-}}=A_{j 1^{1}}^{\alpha_{11}} \cdots A_{j_{s}}^{\alpha_{s}}
$$

Proof. Choose $P \in \mathscr{2}^{m \times n}$ as in Lemma 1.4. Let $V=$ $\left\{\left(\beta_{1}, \cdots, \beta_{n}\right)^{T} \mid\left(\beta_{1}, \cdots, \beta_{n}\right)^{T} \in \mathbf{R}^{n+1}, P\left(\beta_{1}, \cdots, \beta_{n}\right)^{T}=0\right\} .\left(\epsilon_{1}, \cdots, \epsilon_{n}\right)^{T} \in V$ and so $V \neq\{0\}$. Let

$$
W=\left\{\left(\beta_{1}, \cdots, \beta_{n}\right)^{T} \mid\left(\beta_{1}, \cdots, \beta_{n}\right)^{T} \in \mathscr{Q}^{n \times 1}, P\left(\beta_{1}, \cdots, \beta_{n}\right)^{T}=0\right\} .
$$

Let $\mu=n-\operatorname{rank}$ of $P$. Then $\operatorname{dim} V$ over $\mathbf{R}=\mu=\operatorname{dim} W$ over 2. Since $V \neq\{0\}$, we have $\mu>0$. $W$ has a basis $H_{1}, \cdots, H_{\mu}$ over 2. Let $H=$ the $n \times \mu$ matrix $\left[H_{1}, \cdots, H_{\mu}\right]$. Then rank of $H=\mu$. So $H_{1}, \cdots, H_{\mu}$ are also linearly independent over $\mathbf{R}$. Hence $H_{1}, \cdots, H_{\mu}$ form a basis of $V$ and of course $H_{1}, \cdots, H_{\mu} \in \mathscr{2}^{n \times 1}$. So there exist $\delta_{1}, \cdots, \delta_{\mu} \in \mathbf{R} \quad$ such that $\left(\epsilon_{1}, \cdots, \epsilon_{n}\right)^{T}=\delta_{1} H_{1}+\cdots+\delta_{\mu} H_{\mu}$. Let $\gamma_{1}, \cdots, \gamma_{\mu} \in \mathcal{Q}$ and set $\left(\alpha_{1}, \cdots, \alpha_{n}\right)^{T}=\gamma_{1} H_{1}+\cdots+\gamma_{\mu} H_{\mu}$. Then clearly $\left(\alpha_{1}, \cdots, \alpha_{n}\right)^{T} \in W$. Also

$$
\sqrt{\sum_{k=1}^{n}\left|\alpha_{k}-\epsilon_{k}\right|^{2}} \leqq \sum_{p=1}^{\mu}\left|\delta_{p}-\gamma_{p}\right|\left\|H_{p}\right\|
$$

Thus for any $\delta \in \mathbf{R}^{+}$we can choose $\left|\delta_{p}-\gamma_{p}\right|, p=1, \cdots, \mu$, small enough so that $\left|\alpha_{k}-\epsilon_{k}\right|<\delta / n, k=1, \cdots, n$. For $\delta$ small enough we then also have $\alpha_{k} \in \mathscr{Q}^{+}, k=1, \cdots, n$. This proves the lemma.

THEOREM 1.6. Let $\left\{w_{1}, w_{2}\right\}$ be a word equation in variables $x_{1}, \cdots, x_{n}$. Let $\left(a_{1}, \cdots, a_{n}\right)$ be a solution of $\left\{w_{1}, w_{2}\right\}$ in $\mathscr{F}_{\mathbf{R}}(\Gamma)$. Then for each $\epsilon \in \mathbf{R}^{+}$, there exists a solution $\left(b_{1}, \cdots, b_{n}\right)$ of $\left\{w_{1}, w_{2}\right\}$ in $\mathscr{F}_{2}(\Gamma)$ such that $\sum_{\imath=1}^{n} d\left(a_{\imath}, b_{\imath}\right)<\epsilon$. 
Proof. Let $a_{i}=A_{i 1}^{\beta_{11}} \cdots A_{i m_{i}}^{\beta_{i m}}$ in standard form, $i=1, \cdots, n$. Let $w_{1}$ start with $x_{t}$ and let $w_{2}$ start with $x_{j}$. Then correspondingly we have

$$
A_{t 1}^{\beta_{t 1}} \cdots=A_{j 1}^{\beta_{11}} \cdots .
$$

Choose $\alpha_{t k} \in \mathscr{Q}^{+}, i=1, \cdots, n, 1 \leqq k \leqq m_{l}$. Let $b_{\imath}=A_{i 1}^{\alpha_{1}} \cdots A_{i m_{i}}^{\alpha_{i} m_{i}}, i=$ $1, \cdots, n$. Then $b_{1}, \cdots, b_{n} \in \mathscr{F}_{2}(\Gamma)$. Also, $w_{1}\left(b_{1}, \cdots, b_{n}\right)=w_{2}\left(b_{1}, \cdots, b_{n}\right)$ if and only if

$$
A_{t 1}^{\alpha_{t 1}} \cdots=A_{11}^{\alpha_{11}} \cdots \text {. }
$$

But by Lemma 1.5 we can choose $\alpha_{t k}$ 's so that (4) holds and $\left|\alpha_{t k}-\beta_{t k}\right|<\epsilon$ for all relevant $i$ and $k$. So clearly $\sum_{i=1}^{n} d\left(a_{i}, b_{i}\right)=\sum_{i, k}\left|\alpha_{i k}-\beta_{i k}\right| \leqq M \epsilon$ where $M=\sum_{i=1}^{n} e\left(a_{i}\right)$. This proves the theorem.

Lemma 1.7. Let $A_{1}, \cdots, A_{n} \in \Gamma, \quad \Lambda \subseteq \Gamma$. Suppose $\alpha_{1}, \cdots, \alpha_{n}$, $\beta_{1}, \cdots, \beta_{n} \in \mathbf{R}^{+}, i_{1}, \cdots, i_{r}, j_{1}, \cdots, j_{s} \in\{1, \cdots, n\}$ such that $A_{l_{1}}^{\alpha_{\alpha_{1}}} \cdots A_{i_{r}}^{\alpha_{i_{r}}}=$

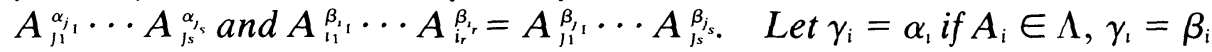

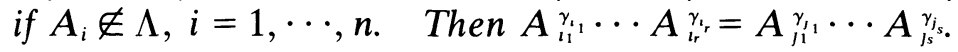

Proof. We prove by induction on $r+s$. Choose $p, q$ maximal such that for $1 \leqq \mu \leqq p, 1 \leqq \nu \leqq q, A_{i_{1}}=A_{i_{\mu}}$ and $A_{l 1}=A_{l v}$. Then

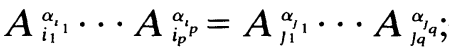

$$
\begin{aligned}
& A_{i 1}^{\beta_{i}} \cdots A_{i_{p} p}^{\beta_{i_{p}}}=A_{j_{1}{ }^{1}}^{\beta_{1}} \cdots A_{j_{q} q_{q}}^{\beta_{i}}
\end{aligned}
$$

Since $A_{i_{\mu}}=A_{j_{v}}$ for $1 \leqq \mu \leqq p, 1 \leqq \nu \leqq q$, we obtain

$$
\boldsymbol{A}_{i_{1}}^{\gamma_{1}} \cdots \boldsymbol{A}_{i_{p}}^{\gamma_{i p}}=\boldsymbol{A}_{j_{1}}^{\gamma_{i_{1}}} \cdots \boldsymbol{A}_{j_{q}}^{\gamma_{q}}
$$

Also, if $p+q<r+s$, then $p<r, q<s$ and

$$
\begin{aligned}
& A_{i_{p+1}}^{\alpha_{i_{p+1}}} \cdots A_{l_{l_{r}}}^{\alpha_{i_{r}}}=A_{j_{q+1}}^{\alpha_{q_{q+1}}} \cdots A_{s_{s}}^{\alpha_{j_{s}}} ; \\
& A_{i_{p}+1}^{\beta_{p_{p+1}}} \cdots A_{l_{r}}^{\beta_{l_{r}}}=A_{j_{q+1}}^{\beta_{q_{q+1}}} \cdots A_{j_{s}}^{\beta_{s_{s}}} .
\end{aligned}
$$

By our induction hypothesis we then also have,

$$
A_{i_{p+1}}^{\gamma_{i_{p}+1}} \cdots A_{i_{r}}^{\gamma_{i_{r}}}=A_{j_{q+1}}^{\gamma_{i_{q}+1}} \cdots A_{j_{s}}^{\gamma_{s^{s}}}
$$

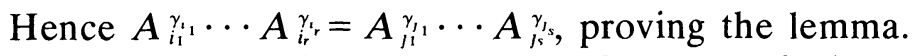

We will need the following refinement of Theorem 1.6.

THEOREM 1.8. Let $\left\{w_{1}, w_{2}\right\}$ be a word equation in variables 
$x_{1}, \cdots, x_{n}$. Let $\left(a_{1}, \cdots, a_{n}\right)$ be a solution of $\left\{w_{1}, w_{2}\right\}$ in $\mathscr{F}_{\mathbf{R}}(\Gamma)$. Then for each $\epsilon \in \mathbf{R}^{+}$, there exists a solution $\left(c_{1}, \cdots, c_{n}\right)$ of $\left\{w_{1}, w_{2}\right\}$ in $\mathscr{F}_{2}(\Gamma)$ such that $\sum_{i=1}^{n} d\left(a_{i}, c_{i}\right)<\epsilon$ and so that for any $A \in \Gamma, A$ appears integrally in each $a_{i}$ implies $A$ appears integrally in each $c_{i}$.

Proof. Let $\Lambda=\{A \mid A \in \Gamma, \quad A$ appears integrally in each $\left.a_{i}\right\}$. Choose $\left(b_{1}, \cdots, b_{n}\right)$ as in Theorem 1.6. Let $a_{t}=A_{t 1}^{\alpha_{i 1}} \cdots A_{i m_{i}}^{\alpha_{i m_{i}}}$, $b_{i}=A_{i 1}^{\beta_{1}} \cdots A_{i m_{i}}^{\beta_{i m_{i}}}, i=1, \cdots, n$ in standard form. Let $\gamma_{i k}=\alpha_{i k}$ if $A_{i k} \in \Lambda$,

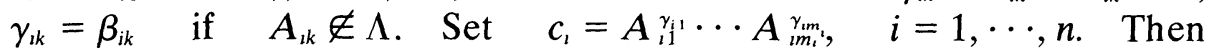
$c_{i} \in \mathscr{F}_{2}(\Gamma), d\left(a_{i}, c_{i}\right) \leqq d\left(a_{i}, b_{i}\right)$. Let $w_{1}$ start with $x_{i}, w_{2}$ start with $x_{j}$. Then correspondingly we have,

$$
\begin{aligned}
& A_{i 1}^{\alpha_{i 1}} \cdots=A_{j 1}^{\alpha_{11}} \cdots \\
& A_{\imath 1}^{\beta_{i 1}} \cdots=A_{11}^{\beta_{11}} \cdots .
\end{aligned}
$$

Then by Lemma 1.7 we also have

$$
A_{i 1}^{\gamma_{11}} \cdots=A_{j 1}^{\gamma_{11}} \cdots
$$

So $w_{1}\left(c_{1}, \cdots, c_{n}\right)=w_{2}\left(c_{1}, \cdots, c_{n}\right)$. This proves the theorem.

Let $\left\{w_{1}, w_{2}\right\}$ be a word equation in variables $x_{1}, \cdots, x_{n}$. A solution $\left(a_{1}, \cdots, a_{n}\right)$ of $\left\{w_{1}, w_{2}\right\}$ in $\mathscr{F}_{\mathbf{R}}(\Gamma)$ is trivial if either there exist $u \in \mathscr{F}_{\mathbf{R}}(\Gamma)$, $k_{1}, \cdots, k_{n} \in Z^{+}$such that $u^{k_{i}}=a_{\imath}, i=1, \cdots, n$, or if there exist $A \in \Gamma$, $\alpha_{1}, \cdots, \alpha_{n} \in \mathbf{R}^{+}$such that $a_{l}=A^{\alpha_{i}}, i=1, \cdots, n$.

THEOREM 1.9. Let $\left\{w_{1}, w_{2}\right\}$ be a word equation in variables $x_{1}, \cdots, x_{n}$. Suppose $\left\{w_{1}, w_{2}\right\}$ has only trivial solutions in any free semigroup. Then $\left\{w_{1}, w_{2}\right\}$ has only trivial solutions in any $\mathscr{F}_{\mathrm{R}}(\Gamma)$.

Proof. Let $\left(a_{1}, \cdots, a_{n}\right)$ be a solution of $\left\{w_{1}, w_{2}\right\}$ in $\mathscr{F}_{\mathbf{R}}(\Gamma)$. By Theorem 1.6, there exist solutions $\left(b_{1}^{(m)}, \cdots, b_{n}^{(m)}\right), m \in Z^{+}$of $\left\{w_{1}, w_{2}\right\}$ in $\mathscr{F}_{2}(\Gamma)$ such that $d\left(a_{i}, b_{i}^{(m)}\right) \rightarrow 0$ as $m \rightarrow \infty, i=1, \cdots, n$. By Theorem 1.1 and our hypothesis, there exist, for each $m \in Z^{+}, u_{m} \in \mathscr{F}_{2}(\Gamma), k(m, i) \in$ $Z^{+}, i=1, \cdots, n$ such that $b_{i}^{(m)}=u_{m}^{k(m, t)}, i=1, \cdots, n$. Now $e\left(b_{t}^{(m)}\right)=e\left(a_{i}\right)$ for all $m \in Z^{+}, i=1, \cdots, n$. If for any $i \in\{1, \cdots, n\}, k(m, i) \rightarrow \infty$, then by Lemma 1.2 (i), $e\left(u_{m}\right)=1$ for some $m \in Z^{+}$. It then follows easily (since $\left.d\left(a_{j}, b_{j}^{(m)}\right)<\infty, j=1, \cdots, n\right)$ that $e\left(a_{j}\right)=1, j=1, \cdots, n$, and $a_{\jmath} \sim a_{r}$ for all $j, r \in\{1, \cdots, n\}$. So we may assume that the $k(m, i)$ 's are bounded for each $i=1, \cdots, n$. So $\left\{(k(m, 1), \cdots, k(m, n)) \mid m \in Z^{+}\right\}$is finite. Hence we can assume without loss of generality (going to a subsequence if necessary) that $k(m, i)=k(t, i)$ for all $m, t \in Z^{+}, i=$ $1, \cdots, n$. Thus there exist $k_{1}, \cdots, k_{n} \in Z^{+}$such that for all $m \in Z^{+}$, $b_{i}^{(m)}=u_{m}^{k_{i}}, i=1, \cdots, n$. If $e\left(u_{m}\right)=1$ for any $m$, then we are done as 
above. So assume $e\left(u_{m}\right)>1$ for all $m \in Z^{+}$. Now for all $m, t \in Z^{+}$, $d\left(b_{1}^{(m)}, b_{1}^{(t)}\right)<\infty$. So $d\left(u_{n}^{k_{1}}, u_{t}^{k_{1}}\right)<\infty$. By Lemma 1.3 (ii), $d\left(u_{m}, u_{t}\right)<$ $\infty$. For $m \in Z^{+}$, let $u_{m}=A_{1}^{\alpha(m, 1)} \cdots A_{r}^{\alpha(m, r)}$ in standard form. For any $\epsilon>0, N \in Z^{+}$, there exist $m, t \in Z^{+}, m, t \geqq N$ such that $d\left(b_{1}^{(m)}, b_{1}^{(t)}\right)<$ $\epsilon$. So by Lemma 1.3 (ii), $d\left(u_{m}, u_{t}\right)<\epsilon$. So for $i=1, \cdots, r,\langle\alpha(m, i)\rangle$ is a Cauchy sequence in $\mathbf{R}^{+}$. Let $\langle\alpha(m, i)\rangle \rightarrow \alpha_{i}$. So $\alpha_{i} \in \mathbf{R}$ $(i=1, \cdots, r)$. Let $a_{1}=B_{1}^{\delta_{1}} \cdots B_{t}^{\delta_{t}}$ in standard form. Then by Lemma 1.3 (i) and the fact that $d\left(a_{1}, u_{m}^{k_{1}}\right) \rightarrow 0$ as $m \rightarrow \infty$, we obtain that $\left\{\alpha_{1}, \cdots, \alpha_{r}\right\} \subseteq\left\{\delta_{1}, \cdots, \delta_{t}\right\}$. Hence $\quad \alpha_{1}, \cdots, \alpha_{r} \in \mathbf{R}^{+}$. Let $u=$ $A_{1}^{\alpha_{1}} \cdots A_{r}^{\alpha_{r}}$. So $u \in \mathscr{F}_{\mathbf{R}}(\Gamma)$ and clearly $d\left(u_{m}, u\right) \rightarrow 0$ as $m \rightarrow \infty$. Let $i \in\{1, \cdots, n\}$. Then by Lemma 1.3(ii), $d\left(u_{m}^{k_{l}}, u^{k_{i}}\right) \leqq k_{l} d\left(u_{m}, u\right)$. So $d\left(u_{m}^{k_{i}}, u^{k_{i}}\right) \rightarrow 0$. Now $d\left(a_{i}, u_{m}^{k_{i}}\right) \rightarrow 0$. Also by Lemma $1.2, d\left(a_{i}, u^{k_{t}}\right) \leqq$ $d\left(a_{i}, u_{m}^{k_{i}}\right)+d\left(u_{m}^{k_{i}}, u^{k_{i}}\right)$ for all $m \in Z^{+}$. So $d\left(a_{l}, u^{k_{t}}\right)=0$ and thus by Lemma 1.2, $a_{l}=u^{k_{i}}, i=1, \cdots, n$. This proves the theorem.

Problem 1.10. Generalize Lentin's theory of principal solutions in the free semigroup [2] to $\mathscr{F}_{\mathbf{R}}$.

2. The semigroup of designs around the unit disc. For $\alpha, \beta \in \mathbf{R}^{+}, \quad \alpha<\beta, \quad$ let $\quad I_{\alpha, \beta}=\left\{x \mid x \in \mathbf{R}^{2}, \alpha<\|x\|<\beta\right\}$. Let $\mathfrak{D}=$ $\left\{(A, \alpha) \mid \alpha \in \mathbf{R}^{+}, \alpha>1, A\right.$ is a closed subset of $\bar{I}_{1, \alpha}$; for all $x \in A$ there exists a sequence $\left\langle x_{n}\right\rangle$ in $A$ such that $x_{n} \rightarrow x$ and $\left\|x_{n}\right\| \neq\|x\|$ for all $n$ \}. For $(A, \alpha) \in \mathfrak{D}$, let $\Phi(A, \alpha)=A$. $\mathfrak{D}$ becomes a semigroup under the following multiplication

$$
(A, \alpha)(B, \beta)=(A \cup \alpha B, \alpha \beta) .
$$

We call $\mathfrak{D}$ the semigroup of designs around the unit disc. The multiplication above is illustrated in Figure 1 . If $(A, \alpha) \in \mathfrak{D}$, then let $l(A, \alpha)=$ $\log \alpha$. So for all $u, v \in \mathfrak{D}, \quad l(u v)=l(u)+l(v)$ and $l(u)>0$. In $\mathfrak{D}^{1}$, set $l(1)=0$.

Remark 2.1. Let $(A, \alpha) \in \mathfrak{D}$. Then $A=\overline{A \cap I_{1, \alpha}}$.

Definition. Let $1 \leqq \beta<\gamma \leqq \alpha . \quad$ Then for $(A, \alpha) \in \mathfrak{D},(A, \alpha)_{[\beta, \gamma]}=$ $(\bar{B}, \gamma / \beta)$ where $B=(1 / \beta)\left(A \cap I_{\beta, \gamma}\right)$. Note that $(A, \alpha)_{[\beta, \gamma]} \in \mathfrak{D}$ and since $A=\bar{A}, \Phi\left((A, \alpha)_{[\beta, \gamma]}\right) \subseteq(1 / \beta) A$. Also we define $(A, \alpha)_{[\beta, \beta]}=1$.

Note that $l\left((A, \alpha)_{[\beta, \gamma]}\right)=\log \gamma-\log \beta . \quad$ Also by Remark 2.1, $(A, \alpha)_{[1, \alpha]}=(A, \alpha)$.

Lemma 2.2. (i) Let $1 \leqq \beta<\gamma<\delta \leqq \alpha, \quad(A, \alpha) \in \mathfrak{D}$. Then $(A, \alpha)_{[\beta, \delta]}=(A, \alpha)_{[\beta, \gamma]}(A, \alpha)_{[\gamma, \delta]}$. 

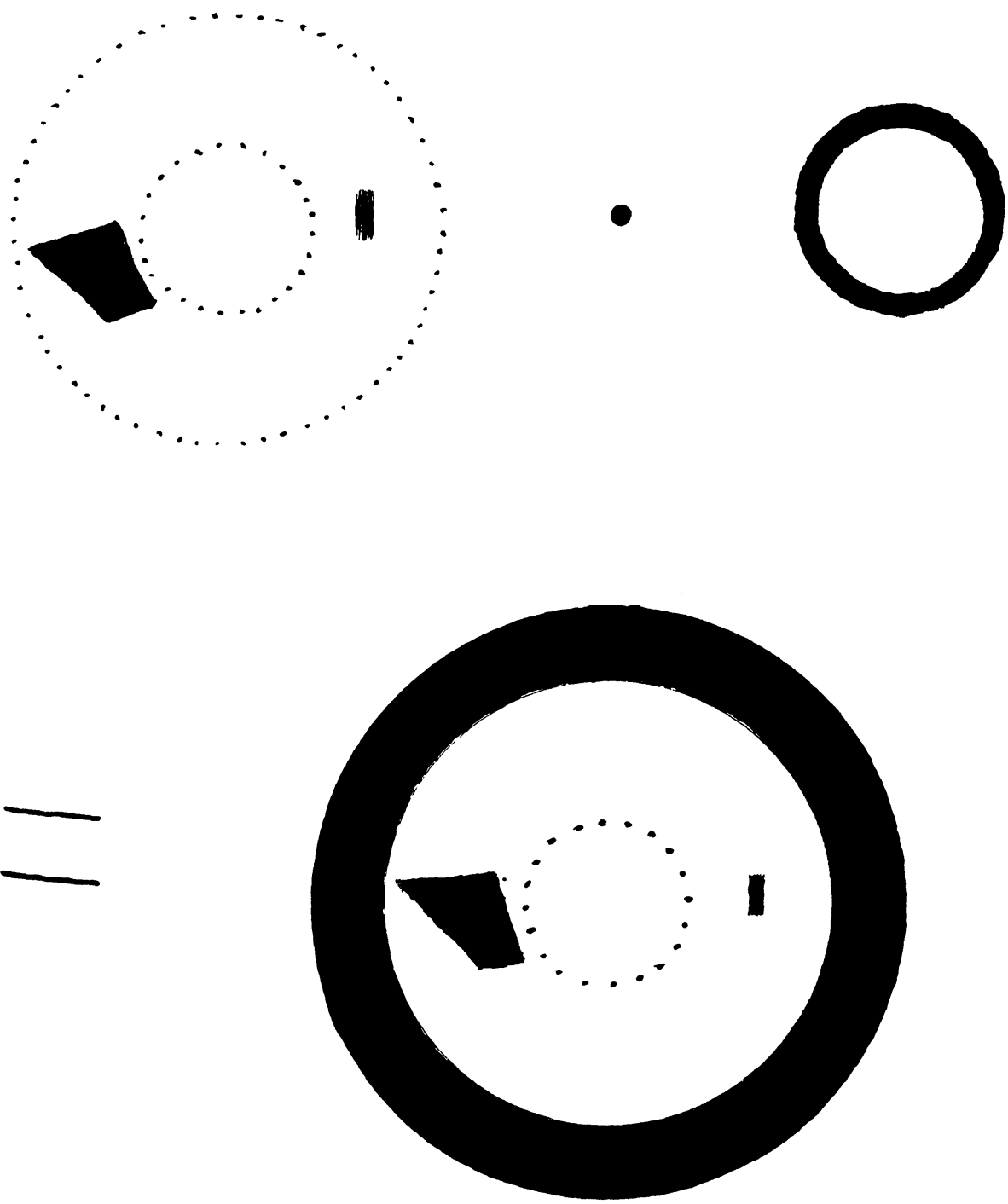

FIGURE 1. Multiplication in $\mathfrak{D}$.

(ii) Let $1 \leqq \beta \leqq \gamma<\delta \leqq \mu \leqq \alpha,(A, \alpha) \in \mathfrak{D}$. Then $l\left((A, \alpha)_{[\gamma, \delta\}}\right) \leqq$ $l\left((A, \alpha)_{[\beta, \mu]}\right)$. Also $l\left((A, \alpha)_{[\gamma, \delta]}\right)=l\left((A, \alpha)_{[\beta, \mu]}\right)$ if and only if $\beta=\gamma$ and $\delta=\mu$.

Proof. (i) Let $x \in A,\|x\|=\gamma$. Then there exists a sequence $\left\langle x_{n}\right\rangle$ of $A$ such that $\left\|x_{n}\right\| \neq \gamma$ for all $n$ and $x_{n} \rightarrow x$. So $A \cap I_{\beta, \delta} \subseteq$ $\left.\overline{\left(A \cap I_{\beta, \gamma}\right.}\right) \cup\left(\overline{A \cap I_{\gamma, \delta}}\right)$. So if $A_{1}=A \cap I_{\beta, \delta}, A_{2}=A \cap I_{\beta, \gamma}, A_{3}=A \cap I_{\gamma, \delta}$, then $\bar{A}_{1}=\bar{A}_{2} \cup \bar{A}_{3}$. Also $\quad(A, \alpha)_{[\beta, \delta]}=\left((1 / \beta) \bar{A}_{1}, \delta / \beta\right), \quad(A, \alpha)_{[\beta, \gamma]}=$ $\left((1 / \beta) \bar{A}_{2}, \gamma / \beta\right)$ and $(A, \alpha)_{[\gamma, \delta]}=\left((1 / \gamma) \bar{A}_{3}, \delta / \gamma\right)$. This yields the result. 
(ii) This follows by noting that by (i), $(A, \alpha)_{[\beta, \mu]}=$ $(A, \alpha)_{[\beta, \gamma]}(A, \alpha)_{[\gamma, \delta]}(A, \alpha)_{[\delta, \mu]}$.

Lemma 2.3. Let $(A, \alpha),(B, \beta) \in \mathfrak{D}$. Set $(C, \gamma)=(A, \alpha)(B, \beta)$. Then $(C, \gamma)_{[1, \alpha]}=(A, \alpha)$ and $(C, \gamma)_{[\alpha, \gamma]}=(B, \beta)$.

Proof. $C=A \cup \alpha B$. So $C \cap I_{1, \alpha} \subseteq A$. It follows that $C \cap I_{1, \alpha}=$ $A \cap I_{1, \alpha} . \quad$ By Remark 2.1, $\Phi\left((C, \gamma)_{[1, \alpha]}\right)=\overline{C \cap I_{1, \alpha}}=\overline{A \cap I_{1, \alpha}}=A$. Thus $(C, \gamma)_{[1, \alpha]}=(A, \alpha)$. Now $C \cap I_{\alpha, \gamma} \subseteq \alpha B$. So $C \cap I_{\alpha, \gamma}=\alpha B \cap I_{\alpha, \gamma}$. Thus $\Phi\left((C, \gamma)_{[\alpha, \gamma]}\right)=(1 / \alpha)\left(C \cap I_{\alpha, \gamma}\right)=(1 / \alpha)\left(\alpha B \cap I_{\alpha, \gamma}\right)=\left(\overline{B \cap I_{1, \beta}}\right)=B$. It follows that $(C, \gamma)_{[\alpha, \gamma]}=(B, \beta)$.

Lemma 2.4. Let $(A, \alpha) \in \mathfrak{D}, 1 \leqq \beta<\gamma \leqq \alpha$ and set $(B, \gamma / \beta)=$ $(A, \alpha)_{[\beta, \gamma]}$. Let $\chi:[1, \gamma / \beta] \rightarrow[\beta, \gamma]$ be the order preserving homeomorphism $\chi(x)=\beta x$. Then for $1 \leqq \delta<\mu \leqq \gamma / \beta,(B, \gamma / \beta)_{[\delta, \mu]}=(A, \alpha)_{[x(\delta), \chi(\mu)]}$.

Proof. $\quad B=(1 / \beta) \overline{\left(A \cap I_{\beta, \gamma}\right.} \subseteq(1 / \beta) A$. So $B \cap I_{\delta, \mu}=I_{\delta, \mu} \cap(1 / \beta) A=$ $(1 / \beta)\left(I_{\chi(\delta), \chi(\mu)} \cap A\right)$. It follows that $\Phi\left((B, \gamma / \beta)_{[\delta, \mu]}\right)=\Phi\left((A, \alpha)_{[\chi(\delta), \chi(\mu)]}\right)$. Also, $\chi(\mu) / \chi(\delta)=\mu / \delta$ and the result follows.

LEMmA 2.5. Let $u_{1}, \cdots, u_{n}, \quad(A, \alpha) \in \mathfrak{D}$ such that $(A, \alpha)=$ $u_{1} \cdots u_{n}$. Then there exist $\alpha_{0}, \cdots, \alpha_{n} \in \mathbf{R}^{+}$such that $1=\alpha_{0}<\alpha_{1}<\cdots<$ $\alpha_{n}=\alpha$ and $(A, \alpha)_{\left[\alpha_{t-1}, \alpha_{i}\right]}=u_{t}, i=1, \cdots, n$.

Proof. Clearly we can assume $n>1$. By Lemma 2.3, there exists $\beta \in(1, \alpha)$ such that $(A, \alpha)_{[1, \beta]}=u_{1},(A, \alpha)_{[\beta, \alpha]}=u_{2} \cdots u_{n}$. We are now done by induction and Lemma 2.4 .

LEMMA 2.6. D is a cancellative semigroup. Let $u_{1}, u_{2}, v_{1}, v_{2} \in \mathfrak{D}$ such that $u_{1} u_{2}=v_{1} v_{2}$. Then exactly one of the following occurs.

(i) $l\left(u_{1}\right)<l\left(v_{1}\right), l\left(v_{2}\right)<l\left(u_{2}\right),\left.u_{1}\right|_{i} v_{1}$ and $\left.v_{2}\right|_{f} u_{2}$.

(ii) $l\left(v_{1}\right)<l\left(u_{1}\right), l\left(u_{2}\right)<l\left(v_{2}\right),\left.v_{1}\right|_{i} u_{1}$ and $\left.u_{2}\right|_{f} v_{2}$.

(iii) $u_{1}=v_{1}$ and $u_{2}=v_{2}$.

Proof. Let $u_{1}, u_{2}, v_{1}, v_{2} \in \mathfrak{D}$ such that $u_{1} u_{2}=v_{1} v_{2}=(A, \alpha)$. By Lemma 2.3 , there exist $\beta, \gamma \in(1, \alpha)$ such that $(A, \alpha)_{[1, \beta]}=u_{1},(A, \alpha)_{[1, \gamma]}=$ $v_{1},(A, \alpha)_{[\beta, \alpha]}=u_{2}$ and $(A, \alpha)_{[\gamma, \alpha]}=v_{2}$. Suppose $l\left(u_{1}\right) \leqq l\left(v_{1}\right)$. Then by Lemma 2.2 (ii), $\beta \leqq \gamma$. So by Lemma 2.2(i), $\left.u_{1}\right|_{\imath} v_{1},\left.v_{2}\right|_{f} u_{2}$. If $l\left(u_{1}\right)=$ $l\left(v_{1}\right)$, then $\beta=\gamma$ and so $u_{1}=v_{1}, u_{2}=v_{2}$. We are now done by symmetry. 
Lemma 2.7. Let $(A, \alpha) \in \mathfrak{D}, x \in A,\|x\|=\beta$. Then,

(i) If $\beta \in(1, \alpha)$, then for $1 \leqq \gamma<\beta<\delta \leqq \alpha, x \in \gamma \Phi\left((A, \alpha)_{[\gamma, \delta]}\right)$.

(ii) If $\beta=1$, then $x \in \Phi\left((A, \alpha)_{[1, \delta]}\right)$ for all $\delta \in(1, \alpha]$.

(iii) If $\beta=\alpha$, then $x \in \gamma \Phi\left((A, \alpha)_{[\gamma, \alpha]}\right)$ for all $\gamma \in[1, \alpha)$.

Proof. (i) $x \in A \cap I_{\gamma, \delta} \subseteq \gamma \Phi\left((A, \alpha)_{[\gamma, \delta]}\right)$.

(ii) There exists a sequence $\left\langle x_{n}\right\rangle$ in $A,\left\|x_{n}\right\| \neq 1$ for all $n$ such that $x_{n} \rightarrow x$. So $x \in \overline{A \cap I_{1, \delta}}=\Phi\left((A, \alpha)_{[1, \delta]}\right)$.

(iii) There exists a sequence $\left\langle x_{n}\right\rangle$ in $A,\left\|x_{n}\right\| \neq \alpha$ for all $n$ such that $x_{n} \rightarrow x$. So $x \in \overline{A \cap I_{\gamma, \alpha}}=\gamma \Phi\left((A, \alpha)_{[\gamma, \alpha]}\right)$.

Definition. Let $U=\left\{x \mid x \in \mathbf{R}^{2},\|x\|=1\right\}$.

(1) Let $K=\bar{K} \subseteq U$. Then for $\alpha \in \mathbf{R}^{+}, \alpha>1$, let $K^{(\alpha)}=(A, \alpha)$ where $\quad A=\{\gamma x \mid x \in K, \quad \gamma \in[1, \alpha]\}$. Let $\mathscr{L}=\left\{K^{(\alpha)} \mid K=\bar{K} \subseteq\right.$ $\left.U, \alpha \in \mathbf{R}^{+}, \alpha>1\right\}$. Then $\mathscr{L} \subseteq \mathfrak{D}$. Note that $K=U \cap \Phi\left(K^{(\alpha)}\right)$. So if $K^{(\alpha)}, L^{(\beta)} \in \mathscr{L}$ and $K^{(\alpha)}=L^{(\beta)}$, then $K=L$ and $\alpha=\beta$. Examples of elements of $\mathscr{L}$ are given in Figure 2.

(2) Let $K^{(\alpha)} \in \mathscr{L}$. Then for $\beta \in \mathbf{R}^{+},\left(K^{(\alpha)}\right)^{\beta}=K^{\left(\alpha^{\beta}\right)}$. This is well defined and agrees with the semigroup definition of power if $\beta \in Z^{+}$.

(3) Let $u, v \in \mathfrak{D}$. Define $u \sim v$ if either there exist $a \in \mathfrak{D}$, $i, j \in Z^{+}$such that $u=a^{i}, v=a^{\prime}$, or if $u, v \in \mathscr{L}$ and $v=u^{\alpha}$ for some $\alpha \in \mathbf{R}^{+}$.

Remark 2.8. (i) $K^{(\alpha)}, K^{(\beta)} \in \mathscr{L}$. Then $K^{(\alpha)} K^{(\beta)}=K^{(\alpha \beta)}$.

(ii) Let $u \in \mathscr{L}, \beta, \gamma \in \mathbf{R}^{+}$. Then $\left(u^{\beta}\right)^{\gamma}=u^{\beta \gamma}, u^{\beta+\gamma}=u^{\beta} u^{\gamma}$ and $l\left(u^{\beta}\right)=\beta l(u)$.

(iii) Let $u \in \mathscr{L}$. Then there exists unique $v \in \mathscr{L}$ such that $u \sim v$ and $l(v)=1$. If $l(u)=\gamma$, then $v^{\gamma}=u$.

(iv) Let $u \in \mathfrak{D}, v \in \mathscr{L}$. If $u \mid v$, then $u \in \mathscr{L}$ and $u \sim v$.

(v) $\sim$ is clearly an equivalence relation on $\mathscr{L}$. If $u \in \mathfrak{D}, v \in \mathscr{L}$, $u \sim v$, then $u \in \mathscr{L}$. It will follow from Theorem 3.16 that $\sim$ is in fact an equivalence relation on $\mathfrak{D}$.

THEOREM 2.9. Let $T$ be a nonempty finite set. For $i \in T, j \in Z^{+}$, choose $u_{i, j} \in \mathfrak{D}$ such that $u_{i, j+1} \mid u_{i, j}$ for all $i \in T, j \in Z^{+}$; and $l\left(u_{i, j}\right) \rightarrow 0$ as $j \rightarrow \infty$ for any fixed $i \in T$. Let $(A, \alpha) \in \mathfrak{D}$. Assume that for each $\beta \in(1, \alpha), j \in Z^{+}$, there exist $k \in Z^{+}, \gamma, \delta \in[1, \alpha], i, p, q \in T$ such that $\gamma<\beta<\delta, k>j$ and so that either $(A, \alpha)_{[\gamma, \delta]}=u_{i, k}$ or else $(A, \alpha)_{[\gamma, \beta]}=u_{p, k}$ and $(A, \alpha)_{[\beta, \delta]}=u_{q, k}$. Then some $u_{i, j} \in \mathscr{L}$.

Proof. Let $U=\left\{x \mid x \in \mathbf{R}^{2},\|x\|=1\right\}$. Let $|T|=n$. We prove by induction on $n$. So assume that the theorem is true for nonempty sets of order less than. $n$ (possibly none). We assume that the conclusion of the 

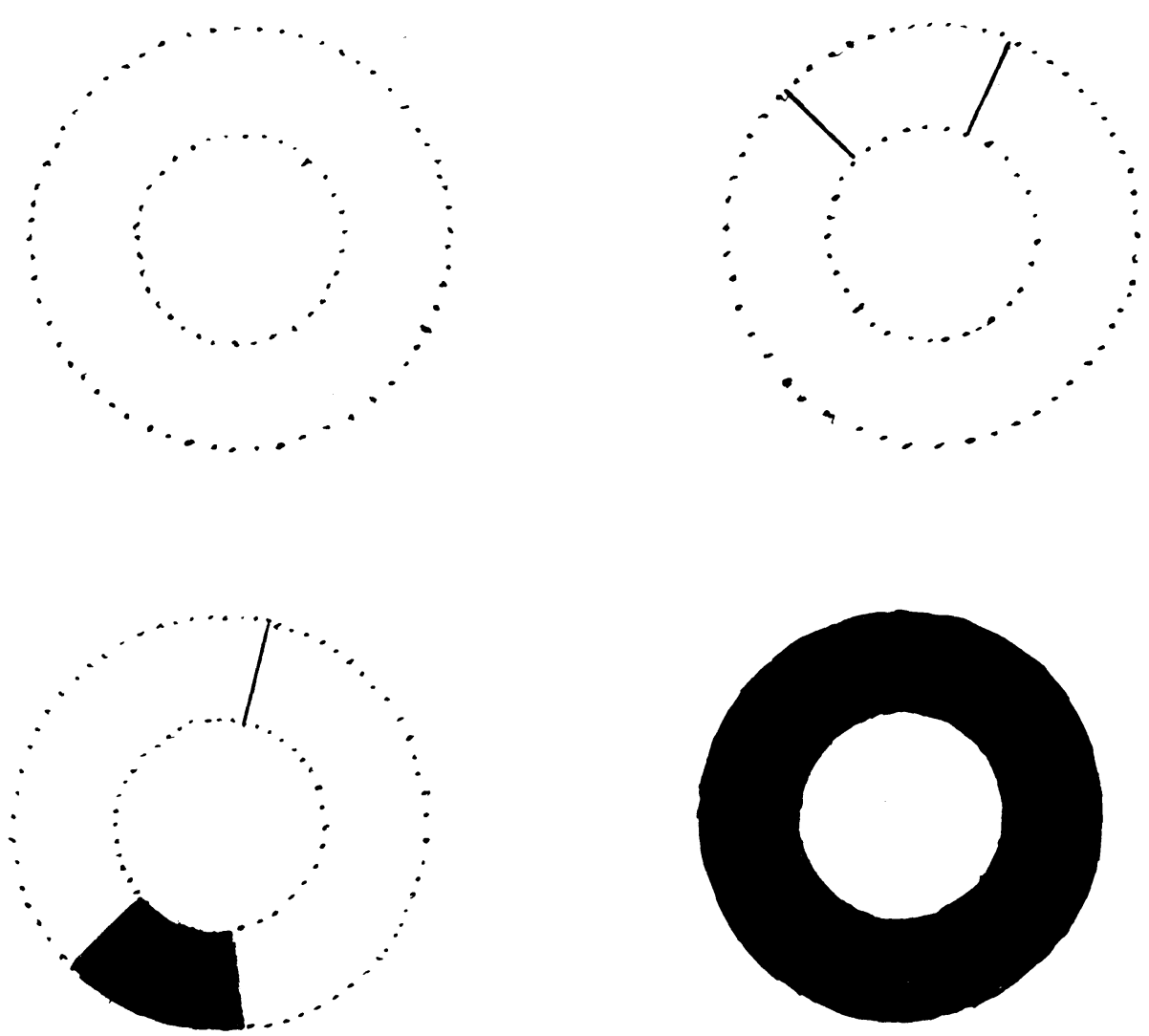

Figure 2. Examples of elements of $\mathscr{L}$.

theorem is false and obtain a contradiction. For $x \in U$, let $P_{x}=$ $\left\{\gamma x \mid \gamma \in \mathbf{R}^{+}\right\}$and $J_{x}=P_{x} \cap I_{1, \alpha}$. Then $\bar{J}_{x}=P_{x} \cap \bar{I}_{1, \alpha}$. First we claim that it suffices to show that for each $x \in U, J_{x} \subseteq A$ or $J_{x} \cap A=\varnothing$. In such a case, first let $J_{x} \subseteq A$. Then since $A$ is closed, $\bar{J}_{x} \subseteq A$. Next let $J_{x} \cap A=\varnothing$. We claim that $\bar{J}_{x} \cap A=\varnothing$. For, let $y \in \bar{J}_{x} \cap A$. Then $\|y\|=1$ or $\alpha$. So there exists a sequence $\left\langle y_{n}\right\rangle$ in $A \cap I_{1, \alpha}$ such that $y_{n} \rightarrow y$. Let $y_{n}=r_{n} x_{n}, r_{n} \in(1, \alpha), x_{n} \in U$. Then $x_{n} \rightarrow x$. Since $y_{n} \in$ $J_{x_{n}} \cap A$, we obtain $J_{x_{n}} \subseteq A$. So $((\alpha+1) / 2) x_{n} \in A$ for all $n$. Since $A$ is closed and $x_{n} \rightarrow x$, we get $((\alpha+1) / 2) x \in A$, contradicting the fact that $J_{x} \cap A=\varnothing$. We have thus shown that for all $x \in U, \bar{J}_{x} \cap A=\varnothing$ or $\bar{J}_{x} \subseteq A$. So letting $K=A \cap U$ we see that $K$ is closed and that $(A, \alpha)=K^{(\alpha)} \in \mathscr{L}$. Then of course some $u_{i, l} \in \mathscr{L}$, a contradiction. This establishes our claim.

So let $x \in U$ such that $J_{x} \not \subset A$. Then $J_{x} \backslash A$ is nonempty and open in $J_{x}$. So there exist $\beta, \gamma \in(1, \alpha)$ such that $\beta<\gamma$ and $\bar{I}_{\beta, \gamma} \cap J_{x} \subseteq$ $J_{x} \backslash A$. Let $\delta \in(\beta, \gamma)$ and let $j \in Z^{+}$. Then there exist $k \in Z^{+}, \mu, \nu \in$ $[1, \alpha], i, p, q \in T$ such that $\mu<\delta<\nu, k>j$ and so that either $(A, \alpha)_{[\mu, \nu]}=$ 
$u_{i, k}$ or else $(A, \alpha)_{[\mu, \delta]}=u_{p, k}$ and $(A, \alpha)_{[\delta, \nu]}=u_{q, k}$. If $j$ is large enough (and hence $l\left(u_{t, k}\right), l\left(u_{p, k}\right), l\left(u_{q, k}\right)$ small enough), we obtain that $\mu, \nu \in(\beta, \gamma)$. Hence by Lemma $2.4,(A, \alpha)_{[\beta, \gamma]}$ satisfies the hypothesis of the theorem for the same $T$. We now claim that for each $i \in T$, there exists $j \in Z^{+}$, such that $u_{\imath, j} \mid(A, \alpha)_{[\beta, \gamma]}$. Suppose not. Then for any $j \in Z^{+}, u_{l, j}$ doesn't come into consideration in the above argument. So $n>1$ and $(A, \alpha)_{[\beta, \gamma]}$ satisfies the theorem with $T \backslash\{i\}$ in place of $T$. So by our induction hypothesis some $u_{p, j} \in \mathscr{L}$, a contradiction. So our claim is established. Since $u_{i, j+1} \mid u_{i, j}$ for all relevant $i$, $j$, we see that there exists $r \in Z^{+}$such that for all $i \in T, j \in Z^{+}, j>r, u_{i, j} \mid(A, \alpha)_{[\beta, \gamma]}$.

We now assume $J_{x} \cap A \neq \varnothing$ and obtain a contradiction. So let $a \in J_{x} \cap A,\|a\|=\delta$. So $\delta \in(1, \alpha)$. There exist $k \in Z^{+}, \mu, \nu \in[1, \alpha]$, $i, p, q \in T$ such that $\mu<\delta<\nu, k>r$ and so that either $(A, \alpha)_{[\mu, \nu]}=u_{i, k}$ or else $(A, \alpha)_{[\mu, \delta]}=u_{p, k}$ and $(A, \alpha)_{[\delta, \nu]}=u_{q, k}$. But $u_{i, k}, u_{p, k}, u_{q, k} \mid(A, \alpha)_{[\beta, \gamma]}$. So in any case $(A, \alpha)_{[\mu, \delta]} \mid(A, \alpha)_{[\beta, \gamma]}$ and $(A, \alpha)_{[\delta, \nu]} \mid(A, \alpha)_{[\beta, \gamma]}$. By Lemma 2.5, there exist $\xi_{1}, \xi_{2} \in \mathbf{R}^{+} \quad$ such that $\xi_{1} \Phi\left((A, \alpha)_{[\mu, \delta]}\right) \cup \xi_{2} \Phi\left((A, \alpha)_{[\delta, \nu]}\right) \subseteq$ $\Phi\left((A, \alpha)_{[\beta, \gamma]}\right)$. By Lemma 2.7(i), $a \in \mu \Phi\left((A, \alpha)_{[\mu, \nu]}\right)$. Since $(A, \alpha)_{[\mu, \nu]}=$ $(A, \alpha)_{[\mu, \delta]} \cdot(A, \alpha)_{[\delta, \nu]}$, there exists $\xi_{3} \in \mathbf{R}^{+}$such that $a \in \xi_{3} \Phi\left((A, \alpha)_{[\mu, \delta]}\right)$ or $a \in \xi_{3} \Phi\left((A, \alpha)_{[\delta, \nu]}\right)$. So for some $\xi \in \mathbf{R}^{+}, \quad \xi a \in \Phi\left((A, \alpha)_{[\beta, \gamma]}\right)=$ $(1 / \beta)\left(A \cap I_{\beta, \gamma}\right) \subseteq(1 / \beta)\left(A \cap \bar{I}_{\beta, \gamma}\right)$. So $\beta \xi a \in A \cap \bar{I}_{\beta, \gamma}$. But $a \in J_{x}$ and so $\beta \xi a \in P_{x}$. But $\|\beta \xi a\| \in[\beta, \gamma] \subseteq(1, \alpha)$. So $\beta \xi a \in A \cap J_{x} \cap \bar{I}_{\beta, \gamma}$, contradicting the fact that $\bar{I}_{\beta, \gamma} \cap J_{x} \subseteq J_{x} \backslash A$. This contradiction completes the proof of the theorem.

3. Word equations in $\mathfrak{D}$. Let $\Gamma$ be a nonempty set. Define $\mathscr{F}_{\mathbf{R}}(\Gamma \mid \varnothing)=\mathscr{F}_{\mathbf{R}}(\Gamma)$ and $\mathscr{F}_{\mathbf{R}}(\Gamma \mid \Gamma)=\mathscr{F}(\Gamma)$. If $\Lambda \subseteq \Gamma, \Lambda \neq \varnothing$, $\Lambda \neq \Gamma$, then let $\mathscr{F}_{\mathbf{R}}(\Gamma \mid \Lambda)$ denote the subsemigroup of $\mathscr{F}_{\mathbf{R}}(\Gamma)$ generated by $\mathscr{F}_{\mathbf{R}}(\Gamma \backslash \Lambda)$ and $\mathscr{F}(\Lambda)$. Let $w \in \mathscr{F}_{\mathbf{R}}(\Gamma)$. Then for any $\Lambda \subseteq \Gamma, w \in \mathscr{F}_{\mathbf{R}}(\Gamma \mid \Lambda)$ if and only if each $A \in \Lambda$ appears integrally in $w$.

Let $\varphi: \Gamma \rightarrow \mathfrak{D}, \Lambda \subseteq \Gamma$, such that $\varphi(\Gamma \backslash \Lambda) \subseteq \mathscr{L}$. Then $\varphi$ extends naturally to a homomorphism $\hat{\varphi}: \mathscr{F}_{\mathbf{R}}(\Gamma \mid \Lambda) \rightarrow \mathfrak{D}$. In fact let $w \in$ $\mathscr{F}_{\mathbf{R}}(\Gamma \mid \Lambda), w=A_{1}^{\epsilon_{1}} \cdots A_{n}^{\epsilon_{n}}$ in standard form. So $A_{i} \in \Lambda$ implies $\epsilon_{l} \in$ $Z^{+}$. Define $\hat{\varphi}(w)=\varphi\left(A_{1}\right)^{\epsilon_{1}} \cdots \varphi\left(A_{n}\right)^{\epsilon_{n}}$. This makes sense, since for $u \in \mathscr{L}, \epsilon \in \mathbf{R}^{+}, u^{\epsilon}$ is defined. Using Remark 2.8(ii), it is easily seen that $\hat{\varphi}$ is a homomorphism. We call $\hat{\varphi}$ the natural extension of $\varphi$ to $\mathscr{F}_{\mathbf{R}}(\Gamma \mid \Lambda)$.

Let $\left(u_{1}, \cdots, u_{n}\right)$ be a solution in $\mathscr{F}_{\mathbf{R}}(\Gamma)$ of a word equation $\left\{w_{1}, w_{2}\right\}$. Let $\Lambda=\{A \mid A \in \Gamma, \quad A$ appears integrally in each $\left.u_{1}, \cdots, u_{n}\right\}$. Then $u_{1}, \cdots, u_{n} \in \mathscr{F}_{\mathbf{R}}(\Gamma \mid \Lambda)$. Let $\varphi: \Gamma \rightarrow \mathfrak{D}$ such that $\varphi(\Gamma \backslash \Lambda) \subseteq \mathscr{L}$. Let $\hat{\varphi}$ be the natural extension of $\varphi$. Let $a_{i}=\hat{\varphi}\left(u_{i}\right)$, $i=1, \cdots, n$. Then $\left(a_{1}, \cdots, a_{n}\right)$ is a solution of $\left\{w_{1}, w_{2}\right\}$ in $\mathfrak{D}$. We say that $\left(a_{1}, \cdots, a_{n}\right)$ follows from $\left(u_{1}, \cdots, u_{n}\right)$. 
REMARK 3.1. In the above notation suppose there exists $\Lambda_{1} \subseteq \Gamma$, $\psi: \Gamma \rightarrow \mathfrak{D}$ such that $\psi\left(\Gamma \backslash \Lambda_{1}\right) \subseteq \mathscr{L}$. Let $\hat{\psi}$ be the natural extension of $\psi$ to $\mathscr{F}_{\mathbf{R}}\left(\Gamma \mid \Lambda_{1}\right)$. Suppose $u_{1}, \cdots, u_{n} \in \mathscr{F}_{\mathbf{R}}\left(\Gamma \mid \Lambda_{1}\right) \quad$ and $\quad a_{1}=\hat{\psi}\left(u_{1}\right), \quad i=$ $1, \cdots, n$. Then $\left(a_{1}, \cdots, a_{n}\right)$ follows from $\left(u_{1}, \cdots, u_{n}\right)$. This is because the above implies that $\Lambda_{1} \subseteq \Lambda$ and so $\Gamma \backslash \Lambda \subseteq \Gamma \backslash \Lambda_{1} \subseteq \mathscr{L}$. Also it is clear that the natural extension of $\psi$ to $\mathscr{F}_{\mathbf{R}}(\Gamma \mid \Lambda)$ is the restriction of $\hat{\psi}$ to $\mathscr{F}_{\mathbf{R}}(\Gamma \mid \Lambda)$.

Even though we are only interested in word equations, it will be convenient to introduce the concept of a constrained word equation.

Definition. Let $\quad w_{1}=w_{1}\left(x_{1}, \cdots, x_{n}\right), \quad w_{2}=w_{2}\left(x_{1}, \cdots, x_{n}\right) \in$ $\mathscr{F}\left(x_{1}, \cdots, x_{n}\right)$. Let $T_{1}, \cdots, T_{s}$ denote $s$ disjoint nonempty subsets of $\left\{x_{1}, \cdots, x_{n}\right\}$. Choose $\alpha_{k} \in \mathbf{R}^{+}$corresponding to each $k \in T_{j}, j=$ $1, \cdots, s$. Let $M_{j}=\left\{\left(x_{k}, \alpha_{k}\right) \mid k \in T_{j}\right\}$. We call $\mathscr{A}=\left\{w_{1}, w_{2} ; M_{1}, \cdots, M_{s}\right\}$ a constrained word equation in variables $x_{1}, \cdots, x_{n}$. We allow the possibility that $m=0$, in which case $\mathscr{A}$ is the word equation $\left\{w_{1}, w_{2}\right\}$. If $1 \leqq i \leqq n$ and $i \notin T$, for every $j, 1 \leqq j \leqq s$, then we say that $x_{i}$ is a free variable of $\mathscr{A}$. Otherwise $x_{i}$ is a constrained variable. If $m=0$, then $x_{i}$ is free $(1 \leqq i \leqq n)$. Let $a_{1}, \cdots, a_{n} \in \mathfrak{D}$. Then $\left(a_{1}, \cdots, a_{n}\right)$ is a solution of $\mathscr{A}$ if the following conditions are satisfied.

(1) $w_{1}\left(a_{1}, \cdots, a_{n}\right)=w_{2}\left(a_{1}, \cdots, a_{n}\right)$.

(2) $\left(x_{k}, \alpha_{k}\right) \in M_{\jmath}$ implies that $a_{k} \in \mathscr{L}$ and $l\left(a_{k}\right)=\alpha_{k}, j=1, \cdots, s$.

(3) Let $\left(x_{\imath}, \alpha_{t}\right) \in M_{p},\left(x_{l}, \alpha_{l}\right) \in M_{q}$. Then $a_{i} \sim a_{\jmath}$ if and only if $p=q$.

Similarly if $a_{1}, \cdots, a_{n} \in \mathscr{F}_{\mathbf{R}}(\Gamma)$, then we say that $\left(a_{1}, \cdots, a_{n}\right)$ is a solution of $\mathscr{A}$ if (1), (2) and (3) above are satisfied with $\mathscr{L}$ replaced by $\mathcal{N}(\Gamma)$.

Definition. Let $\mathscr{A}=\left\{w_{1}, w_{2} ; M_{1}, \cdots, M_{s}\right\}$ be a constrained word equation in variables $x_{1}, \cdots, x_{n}$.

(1) Let $\mu=\left(a_{1}, \cdots, a_{n}\right), \nu=\left(b_{1}, \cdots, b_{n}\right)$ be solutions of $\mathscr{A}$ in $\mathfrak{D}, \mathscr{F}_{\mathrm{R}}$ respectively. (Note that then for each constrained variable $x_{i}, l\left(a_{t}\right)=$ $\left.l\left(b_{1}\right)\right)$. Then we say that $\mu$ follows from $\nu$ (as solutions of $\mathscr{A}$ ) if $\mu$ follows from $\nu$ as solutions of the word equation $\left\{w_{1}, w_{2}\right\}$.

(2) A solution $\mu$ of $\mathscr{A}$ in $\mathfrak{D}$ is resolvable if it follows from a solution of $\mathscr{A}$ in $\mathscr{F}_{\mathbf{R}}(\Gamma)$ with $|\Gamma| \leqq r+s \leqq n$ where $r$ is the number of free variables of $\mathscr{A}$.

(3) $\mathscr{A}$ is resolvable in $\mathfrak{D}$ if every solution of $\mathscr{A}$ in $\mathscr{D}$ is resolvable.

Lemma 3.2. Let $w_{1}, w_{2} \in \mathscr{F}\left(x_{1}, \cdots, x_{n}\right)$. Let $a_{1}, \cdots, a_{n} \in \mathcal{N}(\Gamma)$ such that $a_{i} \sim a_{1}$ for all $i, j$. Suppose $l\left(w_{1}\left(a_{1}, \cdots, a_{n}\right)\right)=$ $l\left(w_{2}\left(a_{1}, \cdots, a_{n}\right)\right)$. Then $w_{1}\left(a_{1}, \cdots, a_{n}\right)=w_{2}\left(a_{1}, \cdots, a_{n}\right)$.

Proof. For some $A \in \Gamma, a_{i}=A^{\alpha_{i}}, \quad \alpha_{i}=l\left(a_{\imath}\right), i=1, \cdots, n$. Let 
$l\left(w_{1}\left(a_{1}, \cdots, a_{n}\right)\right)=l\left(w_{2}\left(a_{1}, \cdots, a_{n}\right)\right)=\beta$. Then clearly $w_{1}\left(a_{1}, \cdots, a_{n}\right)=$ $A^{\beta}=w_{2}\left(a_{1}, \cdots, a_{n}\right)$.

Lemma 3.3. Let $a_{1}, \cdots, a_{n} \in \mathscr{L}, b_{1}, \cdots, b_{n} \in \mathcal{N}(\Gamma)$. Suppose that $a_{t} \sim a_{j}$ implies $b_{i} \sim b_{j}$ for $i, j \in\{1, \cdots, n\}$. Assume further that $l\left(a_{i}\right)=$ $l\left(b_{i}\right), i=1, \cdots, n$. Let $w_{1}, w_{2} \in \mathscr{F}\left(x_{1}, \cdots, x_{n}\right)$ such that $w_{1}\left(a_{1}, \cdots, a_{n}\right)=$ $w_{2}\left(a_{1}, \cdots, a_{n}\right)$. Then $w_{1}\left(b_{1}, \cdots, b_{n}\right)=w_{2}\left(b_{1}, \cdots, b_{n}\right)$.

Proof. We prove by induction on length of $w_{1} w_{2}$ in $\mathscr{F}\left(x_{1}, \cdots, x_{n}\right)$. We can assume without loss of generality that each $x_{i}$ appears in $w_{1} w_{2}$. Let $w_{1}=x_{i 1} \cdots x_{i s}, w_{2}=x_{j_{1}} \cdots x_{j i}$. So

$$
a_{i_{1}} \cdots a_{i_{s}}=a_{j_{1}} \cdots a_{j_{t}}=a .
$$

Choose $p, q$ maximal so that $1 \leqq p \leqq s, 1 \leqq q \leqq t$; for $1 \leqq k \leqq p, a_{i 1} \sim a_{i k}$ and for $1 \leqq k \leqq q, a_{j 1} \sim a_{j k}$. Now $\left.a_{i_{1}}\right|_{i} a_{j_{1}}$ or $\left.a_{j_{1} 1}\right|_{i} a_{i_{1}}$. So by Remark 2.8(iv), $a_{i_{1}} \sim a_{j_{1}}$. Let $u=a_{i_{1}} \cdots a_{i_{p}}$ and $v=a_{j_{1}} \cdots a_{j_{q}}$. Then $u, v \in \mathscr{L}$. Also $a=$ $u b=v c$ for some $b, c \in \mathfrak{D}^{1}$. First assume $p=s$. Then $b=1$. If $q \neq t$, then $a_{j q+1} \mid u$ and so $a_{1 q+1} \sim u \sim a_{j 1}$, a contradiction. So $q=t$. Then $a_{i} \sim a_{j}$ for all $i, j$. Hence $b_{i} \sim b_{j}$ for all $i, j$. Since $l\left(b_{i}\right)=l\left(a_{i}\right)$ for all $i$, we obtain that $l\left(w_{1}\left(b_{1}, \cdots, b_{n}\right)\right)=l\left(w_{1}\left(a_{1}, \cdots, a_{n}\right)\right)=l\left(w_{2}\left(a_{1}, \cdots, a_{n}\right)\right)=$ $l\left(w_{2}\left(b_{1}, \cdots, b_{n}\right)\right)$. We are then done by Lemma 3.2. Similarly we are done if $q=t$. So assume $p<s$ and $q<t$. We claim that $u=$ $v$. Otherwise, by symmetry, let $v=u v_{1}, v_{1} \in \mathscr{L}$. Then $b=v_{1} c$. Since $\left.a_{i_{p+1}}\right|_{i} b$, we see that $\left.a_{i_{p+1}}\right|_{i} v_{1}$ or $\left.v_{1}\right|_{i} a_{i_{p+1}}$. So $a_{i_{p+1}} \sim v_{1} \sim a_{i 1}$, a contradiction. So $u=v$ and $b=c$. Thus

$$
a_{i_{1}} \cdots a_{i_{p}}=a_{j 1} \cdots a_{j q} ; a_{t_{p+1}} \cdots a_{t_{s}}=a_{j q+1} \cdots a_{j v} .
$$

By our induction hypothesis,

$$
b_{t 1} \cdots b_{i_{p}}=b_{j_{1}} \cdots b_{j_{q}} \quad \text { and } \quad b_{i_{p+1}} \cdots b_{l_{s}}=b_{j_{q+1}} \cdots b_{j t}
$$

So $b_{i_{1}} \cdots b_{i_{s}}=b_{j_{1}} \cdots b_{t_{t}}$ and we are done.

LeMma 3.4. Let $\mathscr{A}=\left\{w_{1}, w_{2} ; M_{1}, \cdots, M_{s}\right\}$ in variables $x_{1}, \cdots, x_{n}$. Suppose for some $w_{3}, w_{4}, w_{5}, w_{6} \in \mathscr{F}\left(x_{1}, \cdots, x_{n}\right), w_{1}=w_{3} w_{4}, w_{2}=w_{5} w_{6}$ such that $w_{3}$ and $w_{5}$ involve only constrained variables. Let $\left(a_{1}, \cdots, a_{n}\right)$ be a solution of $\mathscr{A}$ in $\mathfrak{D}$. Suppose $w_{3}\left(a_{1}, \cdots, a_{n}\right)=w_{5}\left(a_{1}, \cdots, a_{n}\right)$. Let $\mathscr{B}=\left\{w_{4}, w_{6} ; M_{1}, \cdots, M_{s}\right\}$ in variables $x_{1}, \cdots, x_{n}$. Then $\left(a_{1}, \cdots, a_{n}\right)$ is a solution of $\mathscr{B}$. If $\left(a_{1}, \cdots, a_{n}\right)$ is resolvable as a solution of $\mathscr{B}$, then it is resolvable as a solution of $\mathscr{A}$. 
Proof. Note that the free and constrained variables of $\mathscr{A}$ and $\mathscr{B}$ are the same. Clearly $w_{4}\left(a_{1}, \cdots, a_{n}\right)=w_{6}\left(a_{1}, \cdots, a_{n}\right)$ and so $\left(a_{1}, \cdots, a_{n}\right)$ is a solution of $\mathscr{B}$. Let $\left(b_{1}, \cdots, b_{n}\right)$ be a solution of $\mathscr{B}$ in $\mathscr{F}_{\mathbf{R}}(\Gamma)$ from which $\left(a_{1}, \cdots, a_{n}\right)$ follows. It suffices to show that $w_{1}\left(b_{1}, \cdots, b_{n}\right)=$ $w_{2}\left(b_{1}, \cdots, b_{n}\right)$. Let $x_{j}$ be a variable appearing in $w_{3} w_{5}$. Then $x_{j}$ is constrained and so $a_{j} \in \mathscr{L}, b_{j} \in \mathcal{N}(\Gamma)$ and $l\left(a_{j}\right)=l\left(b_{j}\right)$. For the same reason if $x_{j}, x_{k}$ appear in $w_{3} w_{5}$, then $a_{j} \sim a_{k}$ if and only if $b_{j} \sim b_{k}$. So by Lemma $3.3, w_{3}\left(b_{1}, \cdots, b_{n}\right)=w_{5}\left(b_{1}, \cdots, b_{n}\right)$. Since $\left(b_{1}, \cdots, b_{n}\right)$ is a solution of $\mathscr{B}, \quad w_{4}\left(b_{1}, \cdots, b_{n}\right)=w_{6}\left(b_{1}, \cdots, b_{n}\right)$. So $\quad w_{1}\left(b_{1}, \cdots, b_{n}\right)=$ $w_{2}\left(b_{1}, \cdots, b_{n}\right)$.

LeMmA 3.5. Let $\mathscr{A}=\left\{w_{1}, w_{1} ; M_{1}, \cdots, M_{s}\right\}$ in variables $x_{1}, \cdots, x_{n}$. Then $\mathscr{A}$ is resolvable in $\mathscr{D}$.

Proof. Let $\left(a_{1}, \cdots, a_{n}\right)$ be a solution of $\mathscr{A}$ in $\mathfrak{D}$. Let $c_{l}=a_{i}$ if $x_{i}$ is a free variable, and otherwise let $c_{i} \in \mathscr{L}$ such that $c_{i} \sim a_{i}, l\left(c_{l}\right)=1$. Then for constrained $x_{i}$ we have $a_{i}=c_{i}^{l\left(a_{i}\right)}$. Let $\Gamma=\left\{A_{1}, \cdots, A_{n}\right\}$ where $A_{i}=$ $A_{j}$ if and only if $i=j$ or $x_{i}, x_{j}$ are constrained and $a_{i} \sim a_{j}$. Then $|\Gamma|=r+s$ where $r$ is the number of free variables of $\mathscr{A}$. Let $b_{i}=A_{i}$ if $x_{i}$ is free and otherwise let $b_{i}=A_{i}^{l\left(a_{i}\right)}$. Then $\left(b_{1}, \cdots, b_{n}\right)$ is a solution of $\mathscr{A}$. Let $\Lambda=\left\{A_{i} \mid x_{i}\right.$ is free $\}$. Then $b_{i} \in \mathscr{F}_{\mathbf{R}}(\Gamma \mid \Lambda), i=1, \cdots, n$. Let $\varphi: \Gamma \rightarrow \mathfrak{D}$ be given by $\varphi\left(A_{i}\right)=c_{i}, i=1, \cdots, n$. Then $\varphi$ is well defined and $\varphi(\Gamma \backslash \Lambda) \subseteq \mathscr{L}$. Let $\hat{\varphi}$ be the natural extension of $\varphi$ to $\mathscr{F}_{\mathbf{R}}(\Gamma \mid \Lambda)$. Then $\hat{\varphi}\left(b_{i}\right)=a_{i}, i=1, \cdots, n$. So $\left(a_{1}, \cdots, a_{n}\right)$ follows from $\left(b_{1}, \cdots, b_{n}\right)$.

LEMMA 3.6. Any constrained word equation without free variables is resolvable in $\mathfrak{9}$.

Proof. Let $\mathscr{A}=\left\{w_{1}, w_{2} ; M_{1}, \cdots, M_{s}\right\}$ in variables $x_{1}, \cdots, x_{n}$ with all variables being constrained. Let $\left(a_{1}, \cdots, a_{n}\right)$ be a solution of $\mathscr{A}$ in D. So each $a_{i} \in \mathscr{L}$. Choose $c_{i} \in \mathscr{L}$ so that $c_{t} \sim a_{l}, l\left(c_{t}\right)=1$. So $. a_{i}=c_{\imath}^{l\left(a_{i}\right)}$. Let $\Gamma=\left\{A_{1}, \cdots, A_{n}\right\}$ with $A_{i}=A_{j}$ if and only if $a_{\imath} \sim a_{j}$. So $|\Gamma|=s$. Let $b_{i}=A_{i}^{l\left(a_{1}\right)}, i=1, \cdots, n$. By Lemma $3.3,\left(b_{1}, \cdots, b_{n}\right)$ is a solution of $\mathscr{A}$. Define $\varphi: \Gamma \rightarrow \mathfrak{D}$ by $\varphi\left(A_{i}\right)=c_{l}, i=1, \cdots, n$. Then $\varphi$ is well defined and $\varphi(\Gamma) \subseteq \mathscr{L}$. Let $\hat{\varphi}$ be the natural extension of $\varphi$ to $\mathscr{F}_{\mathbf{R}}(\Gamma)$. Then $\hat{\varphi}\left(b_{t}\right)=a_{t}, \quad i=1, \cdots, n$. So $\left(a_{1}, \cdots, a_{n}\right)$ follows from $\left(b_{1}, \cdots, b_{n}\right)$.

Lemma 3.7. Let $\mathscr{A}=\left\{w_{1}, w_{2} ; M_{1}, \cdots, M_{s}\right\}$ in variables $x_{1}, \cdots, x_{n}$. Let $w_{3} \in \mathscr{F}\left(x_{1}, \cdots, x_{n}\right)$ and let $\mathscr{B}=\left\{w_{3} w_{1}, w_{3} w_{2} ; M_{1}, \cdots, M_{s}\right\}$ in the same variables. Let $\left(a_{1}, \cdots, a_{n}\right)$ be a solution of $\mathscr{B}$. Then $\left(a_{1}, \cdots, a_{n}\right)$ is a solution of $\mathscr{A}$. If $\left(a_{1}, \cdots, a_{n}\right)$ is resolvable as a solution of $\mathscr{A}$, then it is resolvable as a solution of $\mathscr{B}$. 
Proof. This follows by noting that in $\mathfrak{D}$ as well as in any $\mathscr{F}_{\mathbf{R}}(\Gamma)$, the solutions of $\mathscr{A}$ and $\mathscr{B}$ are the same.

Lemma 3.8. Let $\mathscr{A}=\left\{w_{1}, w_{2} ; M_{1}, \cdots, M_{s}\right\}$ in variables $x_{1}, \cdots, x_{n}$. Suppose $x_{1}$ is a free variable not occuring in $w_{1} w_{2}$. Let $\mathscr{B}=$ $\left\{w_{1}, w_{2} ; M_{1}, \cdots, M_{s}\right\}$ in variables $x_{2}, \cdots, x_{n}$. If $\mathscr{B}$ is resolvable in $\mathfrak{D}$, then so is $\mathscr{A}$.

Proof. Let $\left(a_{1}, \cdots, a_{n}\right)$ be a solution of $\mathscr{A}$ in $\mathfrak{D}$. Then $\left(a_{2}, \cdots, a_{n}\right)$ is a solution of $\mathscr{B}$ in $\mathfrak{D}$. So $\left(a_{2}, \cdots, a_{n}\right)$ follows from some solution $\left(b_{2}, \cdots, b_{n}\right)$ of $\mathscr{B}$ in $\mathscr{F}_{\mathbf{R}}(\Gamma)$ with $|\Gamma| \leqq r+s$ where $r$ is the number of free variables of $\mathscr{B}$. Correspondingly there exist $\Lambda \subseteq \Gamma, \varphi: \Gamma \rightarrow \mathfrak{P}$ such that $b_{2}, \cdots, b_{n} \in \mathscr{F}_{\mathbf{R}}(\Gamma \mid \Lambda), \varphi(\Gamma \mid \Lambda) \subseteq \mathscr{L}$ and the natural extension $\hat{\varphi}$ of $\varphi$ to $\mathscr{F}_{\mathbf{R}}(\Gamma \mid \Lambda)$ satisfies $\hat{\varphi}\left(b_{i}\right)=a_{i}, i=2, \cdots, n$. Let $b_{1} \notin \mathscr{F}_{\mathbf{R}}(\Gamma)$ and set $\Gamma_{1}=$ $\Gamma \cup\left\{b_{1}\right\}, \quad \Lambda_{1}=\Lambda \cup\left\{b_{1}\right\}$. Then $\left(b_{1}, \cdots, b_{n}\right)$ is a solution of $\mathscr{A}$ in $\mathscr{F}_{\mathbf{R}}\left(\Gamma_{1}\right)$. Extend $\varphi$ to $\varphi_{1}$ by setting $\varphi_{1}\left(b_{1}\right)=a_{1}$. Then $b_{1}, b_{2}, \cdots, b_{n} \in \mathscr{F}_{\mathbf{R}}\left(\Gamma_{1} \mid \Lambda_{1}\right), \varphi_{1}\left(\Gamma_{1} \backslash \Lambda_{1}\right) \subseteq \mathscr{L}$ and the natural extension $\hat{\varphi}_{1}$ of $\varphi_{1}$ to $\mathscr{F}_{\mathrm{R}}\left(\Gamma_{1} \mid \Lambda_{1}\right)$ satisfies $\hat{\varphi}_{1}\left(b_{1}\right)=a_{l}, i=1, \cdots, n$. So $\left(a_{1}, \cdots, a_{n}\right)$ follows from $\left(b_{1}, \cdots, b_{n}\right),\left|\Gamma_{1}\right| \leqq r+1+s$ and the number of free variables of $\mathscr{A}$ is $r+1$.

Lemma 3.9. Let $\mathscr{A}=\left\{w_{1}, w_{2} ; M_{1}, \cdots, M_{s}\right\}$ in variables $x_{1}, \cdots, x_{n}$. Suppose $\left(a_{1}, \cdots, a_{n}\right)$ is a solution of $\mathscr{A}$ in $\mathfrak{D}$. Assume that for some $i \neq j, x_{i}$ and $x_{j}$ are free variables and $a_{i}=a_{j}$. Let $w_{i}^{\prime}\left(x_{1}, \cdots, x_{n}\right)=$ $w_{t}\left(x_{1}, \cdots, x_{j-1}, x_{i}, x_{j+1}, \cdots, x_{n}\right), t=1,2$. Then $x_{j}$ does not appear in $w_{1}^{\prime} w_{2}^{\prime}$. Let $\mathscr{B}=\left\{w_{1}^{\prime}, w_{2}^{\prime} ; M_{1}, \cdots, M_{s}\right\}$ in variables $x_{1}, \cdots, x_{n}$. If $\mathscr{B}$ is resolvable in $\mathfrak{D}$, then the solution $\left(a_{1}, \cdots, a_{n}\right)$ of $\mathscr{A}$ is resolvable in $\mathfrak{D}$.

Proof. Clearly $\left(a_{1}, \cdots, a_{n}\right)$ is also a solution of $\mathscr{B}$. Let $\left(b_{1}, \cdots, b_{n}\right)$ be a solution of $\mathscr{B}$ in $\mathscr{F}_{\mathrm{R}}(\Gamma)$ from which $\left(a_{1}, \cdots, a_{n}\right)$ follows. Then $\mu=\left(b_{1}, \cdots, b_{l-1}, b_{l}, b_{1+1}, \cdots, b_{n}\right)$ is also a solution of $\mathscr{A}$ and $\left(a_{1}, \cdots, a_{n}\right)$ follows from $\mu$.

LemmA 3.10. Let $\mathscr{A}=\left\{w_{1}, w_{2} ; M_{1}, \cdots, M_{s}\right\}$ in variables $x_{1}, \cdots, x_{n}$. Let $\left(a_{1}, \cdots, a_{n}\right)$ be a solution of $\mathscr{A}$ in $\mathfrak{D}$. Suppose that for some $i, x_{1}$ is free and $a_{i} \in \mathscr{L}$. If $a_{i} \sim a_{1}$ for some $\left(x_{i}, \alpha_{j}\right) \in M_{p}$, then let $M_{p}^{\prime}=$ $M_{p} \cup\left\{\left(x_{i}, l\left(a_{t}\right)\right)\right\}, M_{q}^{\prime}=M_{q}$ for $q \neq p$ and set $\mathscr{B}=\left\{w_{1}, w_{2} ; M_{1}^{\prime}, \cdots, M_{s}^{\prime}\right\}$ in variables $x_{1}, \cdots, x_{n}$. If $a_{i} \neq a_{j}$ for any constrained variable $x_{j}$, then set $\mathscr{B}=\left\{w_{1}, w_{2} ; M_{1}, \cdots, M_{s},\left\{\left(x_{i}, l\left(a_{i}\right)\right)\right\}\right\}$ in variables $x_{1}, \cdots, x_{n}$. Then $\mathscr{B}$ has lesser number of free variables than $\mathscr{A}$. If $\mathscr{B}$ is resolvable in $\mathfrak{D}$ then so is the solution $\left(a_{1}, \cdots, a_{n}\right)$ of $\mathscr{A}$.

Proof. Let $r$ be the number of free variables of $\mathscr{A}$. Then $\mathscr{B}$ has 
$r-1$ free variables. Clearly $\left(a_{1}, \cdots, a_{n}\right)$ is also a solution of $\mathscr{B}$. Let $\left(a_{1}, \cdots, a_{n}\right)$ follow from a solution $\left(b_{1}, \cdots, b_{n}\right)$ of $\mathscr{B}$ in $\mathscr{F}_{\mathbf{R}}(\Gamma)$ with $|\Gamma| \leqq(r-1)+(s+1)=r+s$. Then clearly $\left(b_{1}, \cdots, b_{n}\right)$ is also a solution of $\mathscr{A}$ and hence the result follows.

Lemma 3.11. Let $\mathscr{A}=\left\{w_{1}, w_{2} ; M_{1}, \cdots, M_{s}\right\}$. Let $\mu=\left(a_{1}, \cdots, a_{n}\right)$ be a solution of $\mathscr{A}$ in $\mathfrak{D}$. Suppose $\left(x_{\imath}, \alpha_{\imath}\right) \in M_{k}$. Assume $a_{\imath}=a_{i}^{\prime} a_{\imath}^{\prime \prime}$ for some $a_{i}^{\prime}, a_{i}^{\prime \prime} \in \mathfrak{D}$. Introduce new variables $x_{i}^{\prime}, x_{\imath}^{\prime \prime}$ and set

$$
\begin{aligned}
& w_{t}^{\prime}\left(x_{1}, \cdots, x_{i-1}, x_{i}^{\prime}, x_{i}^{\prime \prime}, x_{i+1}, \cdots, x_{n}\right) \\
& \quad=w_{t}\left(x_{1}, \cdots, x_{i-1}, x_{i}^{\prime} x_{\imath}^{\prime \prime}, x_{i+1}, \cdots, x_{n}\right) \\
& \quad \in \mathscr{F}\left(x_{1}, \cdots, x_{i-1}, x_{\imath}^{\prime}, x_{i}^{\prime \prime}, x_{i+1}, \cdots, x_{n}\right), \quad t=1,2 .
\end{aligned}
$$

Let $M_{j}^{\prime}=M_{i}$ for $j \neq k, M_{k}^{\prime}=\left\{\left(x_{i}^{\prime}, l\left(a_{i}^{\prime}\right)\right),\left(x_{i}^{\prime \prime}, l\left(a_{i}^{\prime \prime}\right)\right)\right\} \cup\left(M_{k} \backslash\left\{\left(x_{i}, \alpha_{i}\right)\right\}\right)$. Let $\mathscr{B}=\left\{w_{1}^{\prime}, w_{2}^{\prime} ; M_{1}^{\prime}, \cdots, M_{s}^{\prime}\right\}$ in variables $x_{1}, \cdots, x_{i-1}, x_{1}^{\prime}, x_{l}^{\prime \prime}, x_{i+1}, \cdots, x_{n}$. Then $\mathscr{B}$ has the same number of free variables as $\mathscr{A}$. Also $\nu=$ $\left(a_{1}, \cdots, a_{i-1}, a_{i}^{\prime}, a_{i}^{\prime \prime}, a_{i+1}, \cdots, a_{n}\right)$ is a solution of $\mathscr{B}$. If $\nu$ is resolvable in $\mathfrak{D}$ then so is $\mu$.

Proof. Let $r$ be the number of free variables of $\mathscr{A}$ (and hence $\mathscr{B})$. First note that since $a_{i}^{\prime}, a_{i}^{\prime \prime} \mid a_{i}, a_{i}^{\prime} \sim a_{i}^{\prime \prime} \sim a_{i}$. It is then obvious that $\nu$ is a solution of $\mathscr{B}$. Let $\nu$ follow from a solution $\left(b_{1}, \cdots, b_{t-1}, b_{i}^{\prime}, b_{i}^{\prime \prime}, b_{i+1}, \cdots, b_{n}\right)$ of $\mathscr{B}$ in $\mathscr{F}_{\mathbf{R}}(\Gamma)$ with $|\Gamma| \leqq r+s$. Let $b_{i}=$ $b_{i}^{\prime} b_{i}^{\prime \prime}$ and let $\xi=\left(b_{1}, \cdots, b_{i-1}, b_{i}, b_{t+1}, \cdots, b_{n}\right)$. It is then clear that $\xi$ is a solution of $\mathscr{A}$ and that $\mu$ follows from $\xi$.

Lemma 3.12. Let $\mathscr{A}=\left\{w_{1}, w_{2} ; M_{1}, \cdots, M_{s}\right\}$ in variables $x_{1}, \cdots, x_{n}$. Let $\mu=\left(a_{1}, \cdots, a_{n}\right)$ be a solution of $\mathscr{A}$ in $\mathfrak{D}$. Suppose $i \neq j, x_{j}$ is a free variable and $a_{j}=a_{1} a_{1}^{\prime}$ for some $a_{j}^{\prime} \in \mathfrak{D}$. Introduce a new variable $x_{j}^{\prime}$. Let

$$
\begin{aligned}
& w_{t}^{\prime}\left(x_{1}, \cdots, x_{l-1}, x_{j}^{\prime}, x_{j+1}, \cdots, x_{n}\right) \\
& \quad=w_{t}\left(x_{1}, \cdots, x_{\jmath-1}, x_{i} x_{l}^{\prime}, x_{j+1}, \cdots, x_{n}\right) \\
& \quad \in \mathscr{F}\left(x_{1}, \cdots, x_{j-1}, x_{\jmath}^{\prime}, x_{\jmath+1}, \cdots, x_{n}\right), \quad t=1,2 .
\end{aligned}
$$

Let $\mathscr{B}=\left\{w_{1}^{\prime}, w_{2}^{\prime} ; M_{1}, \cdots, M_{s}\right\}$ in variables $x_{1}, \cdots, x_{\jmath-1}, x_{j}^{\prime}, x_{j+1}, \cdots, x_{n}$. Then $\nu=\left(a_{1}, \cdots, a_{j-1}, a_{j}^{\prime}, a_{j+1}, \cdots, a_{n}\right)$ is a solution of $\mathscr{B}$. If $\nu$ is resolvable then so is $\mu$.

Proof. Let $r$ be the number of free variables of $\mathscr{A}$ (and hence $\mathscr{B}$ ). It is clear that $\nu$ is a solution of $\mathscr{B}$. Let $\nu$ follow from a solution 
$\left(b_{1}, \cdots, b_{l-1}, b_{j}^{\prime}, b_{l+1}, \cdots, b_{n}\right)$ of $\mathscr{B}$ in $\mathscr{F}_{\mathbf{R}}(\Gamma)$ with $|\Gamma| \leqq r+s$. Let $b_{j}=$ $b_{i} b_{j}^{\prime}$. Then $\delta=\left(b_{1}, \cdots, b_{j-1}, b_{j}, b_{j+1}, \cdots, b_{n}\right)$ is a solution of $\mathscr{A}$ and $\mu$ follows from $\delta$.

Let $r \in \mathbf{N}$ and consider the following:

Every constrained word equation in less than $r$ free

variables (possibly none) is resolvable in $\mathfrak{D}$.

Lemma 3.13. Assume (*). Let $\mathscr{A}=\left\{w_{1}, w_{2} ; \cdots\right\}$ in variables $x_{1}, \cdots, x_{n}$. Assume $\mathscr{A}$ has exactly $r$ free variables and that $w_{1}$ and $w_{2}$ start with different variables, at least one of which is free. Then $\mathscr{A}$ is resolvable in $\mathfrak{D}$.

Proof. Let $\left(a_{1}, \cdots, a_{n}\right)$ be a solution of $\mathscr{A}$ in $\mathfrak{D}$. Assume $\left(a_{1}, \cdots, a_{n}\right)$ is not resolvable. We will obtain a contradiction. Let $T=\left\{i \mid x_{\imath}\right.$ is a constrained variable $\}$. So by $(*)$ and Lemma 3.8, each free variable occurs in $w_{1} w_{2}$. Let $x_{i}$ appear $m_{i}^{(1)}$ times in $w_{1} w_{2}, i=$ $1, \cdots, n$. Then $m_{i}^{(1)} \in \mathbf{N}$ for $i \in T$ and $m_{i}^{(1)} \in Z^{+}$for $i \notin T$. Let $u=$ $w_{1} w_{2}\left(a_{1}, \cdots, a_{n}\right)$. So $u$ is a word in $a_{1}, \cdots, a_{n}$ with $a_{t}$ appearing $m_{i}^{(1)}$ times, $i=1, \cdots, n$. Now let $\mathscr{A}^{(1)}=\mathscr{A}, w_{1}^{(1)}=w_{1}, w_{2}^{(1)}=w_{2}, x_{1}^{(1)}=x_{i}, a_{i}^{(1)}=$ $a_{i}, i=1, \cdots, n$. We will construct a sequence of constrained word equations $\mathscr{A}^{(k)}=\left\{w_{1}^{(k)}, w_{2}^{(k)} ; \cdots\right\}$ in variables $x_{1}^{(k)}, \cdots, x_{n}^{(k)}$ with solutions $\left(a_{1}^{(k)}, \cdots, a_{n}^{(k)}\right)$ in $\mathfrak{D}$ such that the following properties are true for all $k \in Z^{+}$.

(I) The constrained variables of $\mathscr{A}^{(k)}$ are exactly $x_{\imath}^{(k)}, i \in T$. Also for $i \in T, a_{i}^{(k)}=a_{i}^{(1)}$.

(II) $\quad u$ is a word in $a_{1}^{(k)}, \cdots, a_{n}^{(k)}$ with $a_{i}^{(k)}$ appearing $m_{i}^{(k)}$ times. If $k>1$, then $m_{i}^{(k)} \geqq m_{i}^{(k-1)}, i=1, \cdots, n$ and $\sum_{i=1}^{n} m_{i}^{(k)}>\sum_{i=1}^{n} m_{i}^{(k-1)}$.

(III) If $k>1$, then $a_{i}^{(k-1)}$ is a word in $a_{1}^{(k)}, \cdots, a_{n}^{(k)}, i=1, \cdots, n$.

(IV) If $k>1$, then $\left.a_{i}^{(k)}\right|_{f} a_{i}^{(k-1)}, i=1, \cdots, n$.

(V) $w_{1}^{(k)}$ and $w_{2}^{(k)}$ start with different variables, at least one of which is free.

(VI) $\left(a_{1}^{(k)}, \cdots, a_{n}^{(k)}\right)$ is not resolvable.

Clearly $\mathscr{A}^{(1)}$ satisfies (I) to (VI). We proceed by induction. So having constructed $\mathscr{A}^{(j)}, 1 \leqq j \leqq k$, satisfying (I) to (VI), we proceed to construct $\mathscr{A}^{(k+1)}$. Let $w_{1}^{(k)}=x_{p}^{(k)} \cdots, w_{2}^{(k)}=x_{q}^{(k)} \cdots$. So $p \neq q$ and either $x_{p}$ or $x_{q}$ is free. We have correspondingly

$$
a_{p}^{(k)} \cdots=a_{q}^{(k)} \cdots
$$

First consider the case that $a_{p}^{(k)}=a_{q}^{(k)}$. If both $x_{p}^{(k)}$ and $x_{q}^{(k)}$ are free, then by applying first Lemma 3.9, and then Lemma 3.8 and $(*)$, we see that 
$\left(a_{1}^{(k)}, \cdots, a_{n}^{(k)}\right)$ is resolvable, a contradiction. Next assume $x_{q}^{(k)}$ is constrained. Then $x_{p}^{(k)}$ is free and $a_{p}^{(k)} \in \mathscr{L}$. Then by Lemma 3.10 and $(*),\left(a_{1}^{(k)}, \cdots, a_{n}^{(k)}\right)$ is resolvable, a contradiction. So $l\left(a_{p}^{(k)}\right) \neq l\left(a_{q}^{(k)}\right)$. By symmetry, assume $l\left(a_{p}^{(k)}\right)<l\left(a_{q}^{(k)}\right)$. Then $\left.a_{p}^{(k)}\right|_{i} a_{q}^{(k)}$. First suppose $x_{q}^{(k)}$ is constrained. Then $x_{p}^{(k)}$ is free and $a_{p}^{(k)} \in \mathscr{L}$. We then get a contradiction as above. So $x_{q}^{(k)}$ is free. Now $a_{q}^{(k)}=a_{p}^{(k)} a_{q}^{(k+1)}$ for some $a_{q}^{(k+1)} \in \mathfrak{D}$. Set $a_{i}^{(k+1)}=a_{i}^{(k)}$ for $i \neq q$. Clearly $\left.a_{i}^{(k+1)}\right|_{f} a_{i}^{(k)}, \quad i=$ $1, \cdots, n$. Also since $q \notin T, a_{i}^{(k)}=a_{i}^{(k+1)}$ for $i \in T$. Trivially, each $a_{i}^{(k)}$ is a word in $a_{1}^{(k+1)}, \cdots, a_{n}^{(k+1)}$. So $u$ is a word in $a_{1}^{(k+1)}, \cdots, a_{n}^{(k+1)}$. Let $a_{i}^{(k+1)}$ appear $m_{i}^{(k+1)}$ times in this word. Then $m_{i}^{(k+1)}=m_{i}^{(k)}$ for $i \neq p$ and $m_{p}^{(k+1)}=m_{p}^{(k)}+m_{q}^{(k)} \geqq m_{p}^{(k)}+m_{q}^{(1)}>m_{p}^{(k)}$. So $\sum_{i=1}^{n} m_{i}^{(k+1)}>\sum_{i=1}^{n} m_{i}^{(k)}$. Now the left hand side of (5) must include more than just $a_{p}^{(k)}$ (as $l\left(a_{p}^{(k)}\right)<$ $\left.l\left(a_{q}^{(k)}\right)\right)$. So let the left side of (5) be $a_{p}^{(k)} a_{t}^{(k)} \cdots$. If $t \neq q$, then (5) becomes

$$
a_{t}^{(k+1)} \cdots=a_{q}^{(k+1)} \cdots, \quad t \neq q .
$$

If $t=q$, then (5) becomes

$$
a_{p}^{(k+1)} a_{q}^{(k+1)} \cdots=a_{q}^{(k+1)} \cdots, \quad p \neq q .
$$

Now introduce a new variable $x_{q}^{(k+1)}$ and set $x_{i}^{(k+1)}=x_{i}^{(k)}$ for $i \neq q$. If (6) holds, then correspondingly let $w_{1}^{(k+1)}=x_{t}^{(k+1)} \cdots, w_{2}^{(k+1)}=x_{q}^{(k+1)} \cdots$. If (7) holds, then correspondingly let $w_{1}^{(k+1)}=x_{p}^{(k+1)} x_{q}^{(k+1)} \cdots, w_{2}^{(k+1)}=x_{q}^{(k+1)} \cdots$. Now applying Lemma 3.12 and then Lemma 3.7 we can construct a constrained word equation $\mathscr{A}^{(k+1)}=\left\{w_{1}^{(k+1)}, w_{2}^{(k+1)} ; \cdots\right\}$ in variables $x_{1}^{(k+1)}, \cdots, x_{n}^{(k+1)}$ such that $\left(a_{1}^{(k+1)}, \cdots, a_{n}^{(k+1)}\right)$ is an unresolvable solution of $\mathscr{A}^{(k+1)}$. Also a close examination of the construction shows that the constrained variables of $\mathscr{A}^{(k+1)}$ are exactly $x_{i}^{(k+1)}, i \in T$. This completes the induction step of our construction.

Now by (II), $\sum_{i=1}^{n} m_{i}^{(k)} \rightarrow \infty$ as $k \rightarrow \infty$. So at least one $m_{t}^{(k)} \rightarrow \infty$. So $l\left(a_{t}^{(k)}\right) \rightarrow 0$. Let $K=\left\{i \mid l\left(a_{i}^{(k)}\right) \rightarrow 0\right\}$. By (I), $T \cap K=\varnothing$. There exists $\epsilon \in \mathbf{R}^{+}$such that for $i \notin K, l\left(a_{i}^{(k)}\right)>\epsilon$ for all $k \in Z^{+}$. Choose $k$ large enough so that $l\left(a_{t}^{(k)}\right)<\epsilon$. Let $a=a_{t}^{(k)}$. Then by (III), for all $\alpha \in Z^{+}$, $\alpha>k, a$ is a word in $a_{i}^{(\alpha)}, i \in K$. Let $P_{\alpha}=\left\{a_{i}^{(\alpha)} \mid i \in K\right\}$. Let $a=$ $(A, \xi)$. Then by Lemma 2.5, for each $\alpha \in Z^{+}, \alpha>k$, there exist $\xi_{0}, \cdots, \xi_{m}$ such that $1=\xi_{0}<\xi_{1}<\cdots<\xi_{m}=\xi$ and for $j=1, \cdots, m$, $(A, \xi)_{\left[\xi_{j-1}, \xi_{j}\right]} \in P_{\alpha}$. So we see that the hypothesis of Theorem 2.9 is satisfied. So $a_{i}^{(\alpha)} \in \mathscr{L}$ for some $i \in K, \alpha \in Z^{+}$. Then since $T \cap K=\varnothing$, $x_{i}^{(\alpha)}$ is a free variable of $\mathscr{A}_{i}^{(\alpha)}$. So by Lemma 3.10 and $(*),\left(a_{1}^{(\alpha)}, \cdots, a_{n}^{(\alpha)}\right)$ is resolvable, contradicting (VI). This completes the proof of Lemma 3.13.

THEOREM 3.14. Every constrained word equation is resolvable in $\mathfrak{D}$. 
Proof. Let $r \in \mathbf{N}$ and assume (*). We must show that every constrained word equation with $r$ free variables is resolvable. Let $\mathscr{A}=\left\{w_{1}, w_{2}, \cdots\right\}$ in variables $x_{1}, \cdots, x_{n}$ with $r$ free variables. We prove by induction on length of $w_{1} w_{2}$ in $\mathscr{F}\left(x_{1}, \cdots, x_{n}\right)$ that $\mathscr{A}$ is resolvable. Let $T=\left\{i \mid x_{i}\right.$ is constrained $\}$. Let $\left(a_{1}, \cdots, a_{n}\right)$ be a solution of $\mathscr{A}$ in $\mathfrak{D}$. If $w_{1}$ and $w_{2}$ start with the same variable, then by our induction hypotheses, Lemma 3.7 and Lemma 3.5, we are done. So let $w_{1}, w_{2}$ start with different variables. If some free variable does not appear in $w_{1} w_{2}$ then since $(*)$ holds, we are done by Lemma 3.8. So assume that each free variable occurs in $w_{1} w_{2}$. If either $w_{1}$ or $w_{2}$ starts with a free variable, then we are done by Lemma 3.13. So assume that both $w_{1}$ and $w_{2}$ start with constrained variables. Let $w_{1}=x_{i 1} \cdots x_{t m}$ and $w_{2}=$ $x_{11} \cdots x_{l \cdot}$. Choose $p, q$ maximal so that $1 \leqq p \leqq m, 1 \leqq q \leqq t$ and for $1 \leqq \alpha \leqq p, 1 \leqq \beta \leqq q$ we have $i_{\alpha}, j_{\beta} \in T$. Clearly,

$$
a_{i_{1}} \cdots a_{i_{m}}=a_{j 1} \cdots a_{j \cdot}
$$

By symmetry assume that $l\left(a_{i_{1}} \cdots a_{i_{p}}\right) \leqq l\left(a_{j 1} \cdots a_{j_{q}}\right)$. Choose $\alpha$ minimal such that $1 \leqq \alpha \leqq q$ and $l\left(a_{i_{1}} \cdots a_{i_{p}}\right) \leqq l\left(a_{j_{1}} \cdots a_{j_{\alpha}}\right)$. Then $a_{j_{\alpha}}=a_{j_{\alpha}}^{\prime} a_{j_{\alpha}}^{\prime \prime}$ for some $a_{j_{\alpha}}^{\prime} \in \mathscr{L}, a_{j_{\alpha}}^{\prime \prime} \in \mathscr{L}^{1}$ such that

$$
a_{11} \cdots a_{i_{p}}=\left\{\begin{array}{lll}
a_{11} \cdots a_{j_{\alpha-1}} a_{l_{\alpha}}^{\prime} & \text { if } & \alpha>1 \\
a_{\prime 1}^{\prime} & \text { if } & \alpha=1
\end{array}\right.
$$

First consider the case $a_{j_{\alpha}}^{\prime \prime}=1$. Then $a_{j_{\alpha}}^{\prime}=a_{j_{\alpha}}$ and $a_{i_{1}} \cdots a_{i_{p}}=$ $a_{j 1} \cdots a_{j \alpha}$. Now by (8), $p=m$ if and only if $\alpha=t$ and in such a case we are done by Lemma 3.6. So let $p<m, \alpha<t$. But now we are done by Lemma 3.4 and our induction hypothesis on $l\left(w_{1} w_{2}\right)$ in $\mathscr{F}\left(x_{1}, \cdots, x_{n}\right)$.

So we are left with the case $a_{j_{\alpha}}^{\prime \prime} \neq 1$. Then $p<m$ and $x_{i_{p+1}}$ is free. Also by (8), (9) we have

$$
a_{t_{p+1}} \cdots=a_{J_{\alpha}}^{\prime \prime} \cdots
$$

Now as in Lemma 3.11 introduce new variables $x_{j_{\alpha}}^{\prime}, x_{j_{\alpha} \text {. }}^{\prime \prime}$ Corresponding to (10), let $w_{1}^{\prime}=x_{i_{p+1}} \cdots$ and $w_{2}^{\prime}=x_{j_{\alpha}}^{\prime \prime} \cdots$. Now an application of Lemma 3.11 followed by Lemma 3.4 (because of (9)) yields a constrained word equation $\mathscr{B}=\left\{\boldsymbol{w}_{1}^{\prime}, \boldsymbol{w}_{2}^{\prime}, \cdots\right\}$ with same free variables as $\mathscr{A}$ (though the total number of variables is $n+1)$ such that (10) represents a solution of $\mathscr{B}$ and the resolvability of $\mathscr{B}$ implies the resolvability of $\left(a_{1}, \cdots, a_{n}\right)$. Also in this construction, $x_{i_{p+1}}$ is free and $x_{j_{\alpha}}^{\prime \prime}$ is constrained. So by Lemma 3.13, $\mathscr{B}$ is resolvable. So $\left(a_{1}, \cdots, a_{n}\right)$ is resolvable and our proof of Theorem 3.14 is complete. 
COROllaRy 3.15. Every word equation is resolvable in $\mathfrak{D}$.

Let $\left\{w_{1}, w_{2}\right\}$ be a word equation in variables $x_{1}, \cdots, x_{n}$. A solution $\left(a_{1}, \cdots, a_{n}\right)$ in $\mathfrak{D}$ of $\left\{w_{1}, w_{2}\right\}$ is trivial if either there exist $u \in \mathfrak{D}$, $k_{1}, \cdots, k_{n} \in Z^{+}$such that $a_{i}=u^{k_{i}}, i=1, \cdots, n$ or if there exist $a \in \mathscr{L}$, $\alpha_{1}, \cdots, \alpha_{n} \in \mathbf{R}^{+}$such that $a^{\alpha_{i}}=a_{i}, i=1, \cdots, n$. Then Theorem 1.9 and Corollary 3.15 imply the following.

THEOREM 3.16. Let $\left\{w_{1}, w_{2}\right\}$ be a word equation in variables $x_{1}, \cdots, x_{n}$ having only trivial solutions is any free semigroup. Then $\left\{w_{1}, w_{2}\right\}$ has only trivial solutions in $\mathfrak{D}$.

4. An approximation theorem for $\mathfrak{D}$. For the definition of a pseudo-metric, see for example [5; p. 129]. Consider the following properties for a function $\varphi: \mathfrak{D} \times \mathfrak{D} \rightarrow \mathbf{R}^{+} \cup\{0\}$.

(a) $\varphi$ is a pseudo-metric on $\mathfrak{D}$.

(b) For any $u_{1}, u_{2} \in \mathfrak{D}, \epsilon \in \mathbf{R}^{+}$, there exists $\delta \in \mathbf{R}^{+}$such that for all $v_{1}, v_{2} \in \mathfrak{D}, \varphi\left(u_{i}, v_{i}\right)<\delta, i=1,2$, implies $\varphi\left(u_{1} u_{2}, v_{1} v_{2}\right)<\epsilon$.

(c) For any $u \in \mathscr{L}, \varphi\left(u, u^{\delta}\right) \rightarrow 0$ as $\delta \rightarrow 1$.

If the above hold, then it is easy to see that for all $u_{1}, \cdots, u_{m} \in \mathfrak{D}$, $\epsilon \in \mathbf{R}^{+}$, there exists $\delta \in \mathbf{R}^{+}$such that for any $v_{1}, \cdots, v_{n} \in \mathfrak{D}, \varphi\left(u_{i}, v_{i}\right)<\delta$, $i=1, \cdots, m$ implies $\varphi\left(u_{1} \cdots u_{m}, v_{1} \cdots v_{m}\right)<\epsilon$.

Using Corollary 3.15, Theorems 1.1 and 1.8, we obtain the following

THEOREM 4.1. Let $\varphi$ satisfy (a), (b) and (c) above. Let $\left(a_{1}, \cdots, a_{n}\right)$ be a solution in $\mathfrak{D}$ of a word equation $\left\{w_{1}, w_{2}\right\}$. Then for every $\epsilon \in \mathbf{R}^{+}$, there exists a strongly resolvable solution $\left(b_{1}, \cdots, b_{n}\right)$ of $\left\{w_{1}, w_{2}\right\}$ in $\mathfrak{D}$ such that $\varphi\left(a_{i}, b_{i}\right)<\epsilon, i=1, \cdots, n$.

Definition. Let $\rho$ be the pseudo-metric on compact subsets of $\mathbf{R}^{2}$ given by $\rho(A, B)=m(A \backslash B \cup B \backslash A)$ where $m$ denotes the Lebesgue measure. Let $\lambda$ be pseudo-metric on $\mathfrak{D}$ given by $\lambda((A, \alpha),(B, \beta))=$ $\rho(A, B)+|\alpha-\beta|$.

THEOREM 4.2. Let $\left(a_{1}, \cdots, a_{n}\right)$ be a solution in (D) of a word equation $\left\{w_{1}, w_{2}\right\}$. Then for every $\epsilon \in \mathbf{R}^{+}$, there exists a strongly resolvable solution $\left(b_{1}, \cdots, b_{n}\right)$ of $\left\{w_{1}, w_{2}\right\}$ in $\mathfrak{D}$ such that $\lambda\left(a_{1}, b_{i}\right)<\epsilon, i=1, \cdots, n$.

Proof. By Theorem 4.1 we must show that $\lambda$ satisfies (a), (b) and (c). First note that $\rho$ satisfies the following.

1. $\rho(A \cup B, C \cup D) \leqq \rho(A, C)+\rho(B, D)$.

2. $\rho(\alpha A, A) \rightarrow 0$ as $\alpha \rightarrow 1$ and $A$ is fixed.

Now let $\left(A_{1}, \alpha_{1}\right),\left(A_{2}, \alpha_{2}\right),\left(B_{1}, \beta_{1}\right),\left(B_{2}, \beta_{2}\right) \in \mathfrak{D}$. Then $\left(A_{1}, \alpha_{1}\right)\left(A_{2}, \alpha_{2}\right)=$ 
$\left(A_{1} \cup \alpha_{1} A_{2}, \alpha_{1} \alpha_{2}\right)$ and $\left(B_{1}, \beta_{1}\right)\left(B_{2}, \beta_{2}\right)=\left(B_{1} \cup \beta_{1} B_{2}, \beta_{1} \beta_{2}\right)$. So

$$
\rho\left(A_{1} \cup \alpha_{1} A_{2}, B_{1} \cup \beta_{1} B_{2}\right) \leqq \rho\left(A_{1}, B_{1}\right)+\rho\left(\alpha_{1} A_{2}, \beta_{1} A_{2}\right)+\rho\left(\beta_{1} A_{2}, \beta_{1} B_{2}\right) .
$$

Let $\left(A_{1}, \alpha_{1}\right),\left(A_{2}, \alpha_{2}\right)$ be fixed and suppose $\lambda\left(\left(A_{1}, \alpha_{1}\right),\left(B_{1}, \beta_{1}\right)\right) \rightarrow 0$, $\lambda\left(\left(A_{2}, \alpha_{2}\right),\left(B_{2}, \beta_{2}\right)\right) \rightarrow 0 . \quad$ Then $\quad \rho\left(A_{1}, B_{1}\right) \rightarrow 0, \quad \beta_{1} \rightarrow \alpha_{1}, \quad \beta_{2} \rightarrow \alpha_{2}$, $\rho\left(A_{2}, B_{2}\right) \rightarrow 0$. So $\rho\left(A_{1} \cup \alpha_{1} A_{2}, B_{1} \cup \beta_{1} B_{2}\right) \rightarrow 0$ and $\beta_{1} \beta_{2} \rightarrow \alpha_{1} \alpha_{2}$. Thus $\lambda\left(\left(A_{1}, \alpha_{1}\right)\left(A_{2}, \alpha_{2}\right),\left(B_{1}, \beta_{1}\right)\left(B_{2}, \beta_{2}\right)\right) \rightarrow 0$. This establishes (b). Next let $K=\bar{K} \subseteq U=\left\{x \mid x \in \mathbf{R}^{2},\|x\|=1\right\}, \quad \alpha, \beta \in \mathbf{R}^{+}, \quad 1<\alpha<\beta$. Then $\Phi\left(K^{(\beta)}\right) \backslash \Phi\left(K^{(\alpha)}\right) \subseteq \bar{I}_{\alpha, \beta}$. So for $\alpha$ fixed, $\lambda\left(K^{(\alpha)}, K^{(\beta)}\right) \rightarrow 0$ as $\beta \rightarrow \alpha$. This establishes (c). (a) is of course trivial and the theorem is proved.

5. Word equations of paths. In this section let $n \in Z^{+}$be fixed and let $\mathscr{D}_{1}$ denote the groupoid of paths in $\mathbf{R}^{n}$ mentioned in the problem at the end of [4]. Also let $*, \equiv, f_{[\alpha, \beta]}$ have the same meaning as in [4]. Let $\mathscr{L}_{1}$ denote the set of lines in $\mathscr{D}_{1}$. Let $\mathscr{L}_{1}^{*}=\left\{f * \mid f \in \mathscr{L}_{1}\right\}$ and let $\mathscr{D}_{1}^{*}=\left\{f * \mid f \in \mathscr{D}_{1}\right\}$. So $\mathscr{D}_{1}^{*}$ is a semigroup. We start off with an analogue of Theorem 2.9.

THEOREM 5.1. Let $T$ be a nonempty finite set. For $i \in T, j \in Z^{+}$, choose $f_{l, j} \in \mathscr{D}_{1}$ such that $\left.f_{h, j+1}\right|_{f} f_{i, j}$ for all $i \in T, j \in Z^{+}$and $l\left(f_{i, j}\right) \rightarrow 0$ as $j \rightarrow \infty$ for any fixed $i \in T$. Let $f \in \mathscr{D}_{1}$. Assume that for each $\beta \in[0,1]$, $j \in Z^{+}$, there exist $\alpha, \gamma \in[0,1], i \in T$ such that $\alpha<\gamma, \beta \in[\alpha, \gamma]$ and $f_{[\alpha, \gamma]} \equiv f_{i, j}$. Then some $f_{p, q} \in \mathscr{L}_{1}$.

Proof. The second part of the proof of [4; Theorem 2.1] shows that there exist $\mu, \nu \in[0,1], \mu<\nu$ such that $f_{[\mu, \nu]} \in \mathscr{L}_{1}$. Choose $\beta \in$ $(\mu, \nu)$. For any $j \in Z^{+}$, there exist $\alpha, \gamma \in[0,1], i \in T$ such that $\alpha<\gamma$, $\beta \in[\alpha, \gamma]$ and $f_{[\alpha, \gamma]} \equiv f_{i, j}$. We can choose $j$ big enough (and hence $l\left(f_{i, j}\right)$ small enough) so that we must have $\alpha>\mu, \gamma<\nu$. Then $f_{i, j} \equiv f_{[\alpha, \gamma]} \in \mathscr{L}_{1}$.

For $a \in \mathscr{L}_{1}^{*}, \alpha \in \mathbf{R}^{+}$, let $a^{\alpha}$ denote the line in $\mathscr{L}_{1}^{*}$ in the same direction as $a$ but with length $\alpha l(a)$. Let $u, v \in \mathscr{D}_{1}^{*}$. Then define $u \sim v$ if either there exist $a \in \mathscr{D}_{1}^{*}, i, j \in Z^{+}$such that $u=a^{i}, v=a^{\prime}$ or if $u, v \in \mathscr{L}_{1}^{*}$ and $v=u^{\alpha}$ for some $\alpha \in \mathbf{R}^{+}$. Because of Theorem 5.1, we can repeat $\$ 3$ (including all the definitions) with $\mathfrak{D}$ replaced by $\mathscr{D}_{1}^{*}$ and $\mathscr{L}$ replaced by $\mathscr{L}_{1}^{*}$. We then obtain the following theorem which answers affirmatively a problem posed at the end of [4].

THEOREM 5.2. Every word equation is resolvable in $\mathscr{D}_{1}^{*}$.

Using Theorem 1.9, we now obtain,

THEOREM 5.3. Let $\left\{w_{1}, w_{2}\right\}$ be a word equation which has only 
trivial solutions in any free semigroup. Then $\left\{w_{1}, w_{2}\right\}$ has only trivial solutions in $\mathscr{D}_{1}^{*}$.

For continuous $f:[0,1] \rightarrow \mathbf{R}^{n}$, let $\|f\|=\sup _{t \in[0,1]}\|f(t)\|$. $u, g \equiv v\}$.

Definition. For $u, v \in \mathscr{D}_{1}^{*}$, let $\eta(u, v)=\inf \left\{\|f-g\| \mid f, g \in \mathscr{D}_{1}, f \equiv\right.$

Then $\eta$ can be shown to have the following properties:

(a) $\eta$ is a pseudo-metric on $\mathscr{D}_{1}^{*}$.

(b) For any $u_{1}, u_{2} \in \mathscr{D}_{1}^{*}, \epsilon \in \mathbf{R}^{+}$, there exists $\delta \in \mathbf{R}^{+}$such that for all $v_{1}, v_{2} \in \mathscr{D}_{1}^{*}, \eta\left(u_{i}, v_{1}\right)<\delta, i=1,2$ implies $\eta\left(u_{1} u_{2}, v_{1} v_{2}\right)<\epsilon$.

(c) For any $u \in \mathscr{L}_{1}^{*}, \eta\left(u, u^{\delta}\right) \rightarrow 0$ as $\delta \rightarrow 1$.

As in $\S 4$, Theorems $1.1,1.8$ and 5.2 easily imply the following.

THEOREM 5.4. Let $\left(a_{1}, \cdots, a_{m}\right)$ be a solution in $\mathscr{D}_{1}^{*}$ of a word equation $\left\{w_{1}, w_{2}\right\}$. Then for every $\epsilon \in \mathbf{R}^{+}$, there exists a strongly resolvable solution $\left(b_{1}, \cdots, b_{m}\right)$ of $\left\{w_{1}, w_{2}\right\}$ in $\mathscr{D}_{1}^{*}$ such that $\eta\left(a_{i}, b_{l}\right)<\epsilon, i=1, \cdots, m$.

Note added in the proof. Problem 1.10 has $\cdot r e c e n t l y$ been solved by the author.

\section{REFERENCES}

1. Ju. I. Hmelevskii, Equations in free semigroups, Proc. Steklov Inst. Math., 107 (1971), 270.

2. A. Lentin, Équations dans les monoides libres, Mouton/Gauthier-Villars, 1972.

3. E. S. Ljapin, Semigroups, Amer. Math. Soc. Transl., (1963). Translation of Polugruppy (1960).

4. M. S. Putcha, Word equations of paths, J. Algebra, (to appear).

5. H. L. Royden, Real Analysis, Second Edition, MacMillan, New York, 1968.

Received December 28, 1976. The author was partially supported by NSF grant MCS 76-05784.

North Carolina State University

RALEIGH, NC 27607 


\section{PACIFIC JOURNAL OF MATHEMATICS EDITORS}

ICHARD ARENS (Managing Editor)

niversity of California

os Angeles, CA 90024

\section{. A. BEAUmont}

niversity of Washington

sattle, WA 98105

. C. MOORE

niversity of California

erkeley, CA 94720
J. DUGUNDJI

Department of Mathematics

University of Southern California

Los Angeles, CA 90007

R. FINN AND J. MiLgRAM

Stanford University

Stanford, CA 94305

\section{ASSOCIATE EDITORS}
F. BECKENBACH
B. H. NEUMANN
F. WOLF
K. YosHidA

\section{SUPPORTING INSTITUTIONS}

NIVERSITY OF BRITISH COLUMBIA

UNIVERSITY OF SOUTHERN CALIFORNIA

ALIFORNIA INSTITUTE OF TECHNOLOGY STANFORD UNIVERSITY

NIVERSITY OF CALIFORNIA

ONTANA STATE UNIVERSITY

UNIVERSITY OF HAWAII

NIVERSITY OF NEVADA

UNIVERSITY OF TOKYO

EW MEXICO STATE UNIVERSITY

UNIVERSITY OF UTAH

REGON STATE UNIVERSITY

NIVERSITY OF OREGON

WASHINGTON STATE UNIVERSITY

UNIVERSITY OF WASHINGTON

SAKA UNIVERSITY

AMERICAN MATHEMATICAL SOCIETY

The Supporting Institutions listed above contribute to the cost of publication of this Journal, but they a t owners or publishers and have no responsibility for its contents or policies.

Mathematical papers intended for publication in the Pacific Journal of Mathematics should be in typ Irm or offset-reproduced (not dittoed), double spaced with large margins. Underline Greek letters in re ierman in green, and script in blue. The first paragraph or two must be capable of being used separately as 'nopsis of the entire paper. Items of the bibliography should not be cited there unless absolutely necessary, hich case they must be identified by author and Journal, rather than by item number. Manuscripts, uplicate, may be sent to any one of the four editors. Please classify according to the scheme of Math. Revieu Idex to Vol. 39. All other communications should be addressed to the managing editor, or Elaine Bart Iniversity of California, Los Angeles, California, 90024.

100 reprints are provided free for each article, only if page charges have been substantially pai dditional copies may be obtained at cost in multiples of 50 .

The Pacific Journal of Mathematics is issued monthly as of January 1966. Regular subscription rate: \$72. year (6 Vols., 12 issues). Special rate: $\$ 36.00$ a year to individual members of supporting institutions.

Subscriptions, orders for numbers issued in the last three calendar years, and changes of address should ent to Pacific Journal of Mathematics, 103 Highland Boulevard, Berkeley, California, 94708.

UBLISHED BY PACIFIC JOURNAL OF MATHEMATICS, A NON-PROFIT CORPORATION

Printed at Jerusalem Academic Press, POB 2390, Jerusalem, Israel.

Copyright (C) 1978 Pacific Journal of Mathematics

All Rights Reserved 


\section{Pacific Journal of Mathematics}

\section{Vol. 75, No. $1 \quad$ September, 1978}

Mieczyslaw Altman, General solvability theorems

Denise Amar and Eric Amar, Sur les suites d'interpolation en plusieurs variables ..........................................

Herbert Stanley Bear, Jr. and Gerald Norman Hile, Algebras which satisfy a second order linear partial differential equation ..................

Marilyn Breen, Sets in $R^{d}$ having $(d-2)$-dimensional kernels ............

Gavin Brown and William Moran, Analytic discs in the maximal ideal space

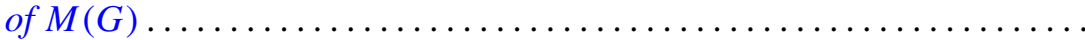

Ronald P. Brown, Quadratic forms with prescribed Stiefel-Whitney

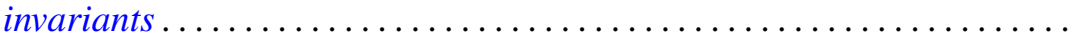

Gulbank D. Chakerian and H. Groemer, On coverings of Euclidean space by convex sets

S. Feigelstock and Z. Schlussel, Principal ideal and Noetherian groups.....

Ralph S. Freese and James Bryant Nation, Projective lattices ............

Harry Gingold, Uniqueness of linear boundary value problems for

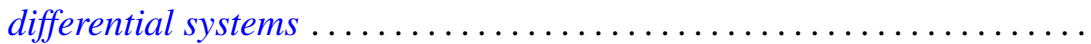

John R. Hedstrom and Evan Green Houston, Jr., Pseudo-valuation domains. . .

William Josephson, Coallocation between lattices with applications to measure extensions

M. Koskela, A characterization of non-negative matrix operators on $l^{p}$ to $l^{q}$ with $\infty>p \geq q>1$

Kurt Kreith and Charles Andrew Swanson, Conjugate points for nonlinear differential equations...........................

Shoji Kyuno, On prime gamma rings ........................ 185

Alois Andreas Lechicki, On bounded and subcontinuous multifunctions ..

Roberto Longo, A simple proof of the existence of modular automorphisms in approximately finite-dimensional von Neumann algebras ...

Kenneth Millett, Obstructions to pseudoisotopy implying isotopy for

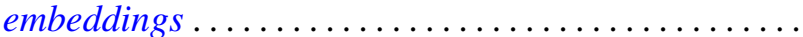

William F. Moss and John Piepenbrink, Positive solutions of elliptic equations. .

Mitsuru Nakai and Leo Sario, Duffin's function and Hadamard's

conjecture

Mohan S. Putcha, Word equations in some geometric semigroups ...

Walter Rudin, Peak-interpolation sets of class $C^{1} \ldots \ldots \ldots$

Elias Saab, On the Radon-Nikodým property in a class of locally convex

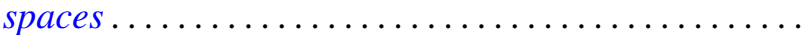

